\title{
Probing highly obscured, self-absorbed galaxy nuclei with vibrationally excited $\mathrm{HCN}^{\star}$
}

\author{
S. Aalto ${ }^{1}$, S. Martín ${ }^{2}$, F. Costagliola ${ }^{3,1}$, E. González-Alfonso ${ }^{4}$, S. Muller ${ }^{1}$, K. Sakamoto ${ }^{5}$, G. A. Fuller ${ }^{6}$, \\ S. García-Burillo ${ }^{7}$, P. van der Werf ${ }^{8}$, R. Neri ${ }^{2}$, M. Spaans ${ }^{9}$, F. Combes ${ }^{10}$, S. Viti ${ }^{11}$, S. Mühle ${ }^{12}$, L. Armus ${ }^{13}$, \\ A. Evans ${ }^{14,15}$, E. Sturm ${ }^{16}$, J. Cernicharo ${ }^{17}$, C. Henkel ${ }^{18,19}$, and T. R. Greve ${ }^{11}$ \\ (Affiliations can be found after the references)
}

Received 25 April 2015 / Accepted 27 July 2015

\begin{abstract}
We present high resolution (0'.4) IRAM PdBI and ALMA mm and submm observations of the (ultra) luminous infrared galaxies ((U)LIRGs) IRAS 17208-0014, Arp220, IC 860 and Zw049.057 that reveal intense line emission from vibrationally excited $\left(v_{2}=1\right) J=3-2$ and $4-3 \mathrm{HCN}$. The emission is emerging from buried, compact $(r<17-70 \mathrm{pc})$ nuclei that have very high implied mid-infrared surface brightness $>5 \times 10^{13} L_{\odot} \mathrm{kpc}^{-2}$. These nuclei are likely powered by accreting supermassive black holes (SMBHs) and/or hot $(>200 \mathrm{~K})$ extreme starbursts. Vibrational, $v_{2}=1$, lines of HCN are excited by intense $14 \mu \mathrm{m}$ mid-infrared emission and are excellent probes of the dynamics, masses, and physical conditions of (U)LIRG nuclei when $\mathrm{H}_{2}$ column densities exceed $10^{24} \mathrm{~cm}^{-2}$. It is clear that these lines open up a new interesting avenue to gain access to the most obscured AGNs and starbursts. Vibrationally excited HCN acts as a proxy for the absorbed mid-infrared emission from the embedded nuclei, which allows for reconstruction of the intrinsic, hotter dust SED. In contrast, we show strong evidence that the ground vibrational state $(v=0)$, $J=3-2$ and 4-3 rotational lines of $\mathrm{HCN}$ and $\mathrm{HCO}^{+}$fail to probe the highly enshrouded, compact nuclear regions owing to strong self- and continuum absorption. The $\mathrm{HCN}$ and $\mathrm{HCO}^{+}$line profiles are double-peaked because of the absorption and show evidence of non-circular motions - possibly in the form of in- or outflows. Detections of vibrationally excited HCN in external galaxies are so far limited to ULIRGs and early-type spiral LIRGs, and we discuss possible causes for this. We tentatively suggest that the peak of vibrationally excited HCN emission is connected to a rapid stage of nuclear growth, before the phase of strong feedback.
\end{abstract}

Key words. galaxies: evolution - galaxies: active - galaxies: nuclei - galaxies: ISM - ISM: molecules

\section{Introduction}

Luminous $\left(L_{\mathrm{IR}}=10^{10}-10^{11} L_{\odot}\right)$ and ultraluminous $\left(L_{\mathrm{IR}} \gtrsim\right.$ $\left.10^{12} L_{\odot}\right)$ infrared galaxies ((U)LIRGS) are powered by either bursts of star formation or AGNs (active galactic nuclei - accreting supermassive black holes (SMBHs)) and are fundamental to galaxy mass assembly over cosmic time (e.g. Elbaz \& Cesarsky 2003; Sanders \& Mirabel 1996). Some (U)LIRGS have deeply embedded nuclei that harbour a very active evolutionary stage of AGNs and starbursts, often with signatures of outflowing and infalling gas (e.g. Banerji et al. 2012; Fabian 1999; González-Alfonso et al. 2012; Silk \& Rees 1998; Spoon et al. 2013). These nuclei are key to understanding nuclear growth and feedback mechanisms and studying them is essential for a complete AGN and starburst census, for constraining orientationbased unification models and fully understanding how galaxies grow and evolve (e.g. Brightman \& Ueda 2012; Merloni et al. 2014).

However, probing inside the optically thick layers of the most enshrouded galaxy nuclei is an observational challenge. X-ray and mid-infrared (mid-IR) surveys are designed to find embedded nuclear activity, but when $A_{\mathrm{v}}>1000 \mathrm{mag}$

\footnotetext{
* Based on observations carried out with the IRAM Plateau de Bure and ALMA Interferometers. IRAM is supported by INSU/CNRS (France), MPG (Germany), and IGN (Spain). ALMA is a partnership of ESO (representing its member states), NSF (USA), and NINS (Japan), together with NRC (Canada) and NSC and ASIAA (Taiwan), in cooperation with the Republic of Chile. The Joint ALMA Observatory is operated by ESO, AUI/NRAO, and NAOJ.
}

$\left(N\left(\mathrm{H}_{2}\right)>10^{24} \mathrm{~cm}^{-2}\right)$, the emission becomes extremely attenuated even at these wavelengths (e.g. Treister et al. 2010; Lusso et al. 2013; Spoon et al. 2002; Roche et al. 2015). Continuum imaging at the more transparent radio- (e.g. Parra et al. 2010) and $\mathrm{mm} /$ sub-mm (e.g. Sakamoto et al. 2008) wavelengths can be used to probe luminosity density and to identify power sources, although interpretations are sometimes complicated by surrounding star formation, free-free absorption, and other optical depth effects. Rotational lines (in the vibrational ground state $v=0$ ) of polar molecules, such as $\mathrm{HCN}$ and $\mathrm{HCO}^{+}$, are common tracers of dense $\left(n>10^{4} \mathrm{~cm}^{-3}\right)$ gas and are often used to probe the nuclear mass, dynamics, and astrochemistry (e.g. Gao \& Solomon 2004; Meijerink \& Spaans 2005) of obscured galaxy nuclei. However, large optical depths and the low-level energies of the transitions (see Fig. 1) make them less well suited to studying deeply embedded, hot, and compact starbursts or obscured AGNs.

In contrast, rotational transitions of vibrationally excited $\left(v_{2}=1\right) \mathrm{HCN}$ occur between energy levels exceeding $1000 \mathrm{~K}$ above ground (Fig. 1) (Ziurys \& Turner 1986). These transitions are usually radiatively excited by the intense mid-IR emission provided by the presence of hot dust and high column densities (see Sect. 2 and Appendix B). Therefore, the dust and gas obscuration impeding the use of other tracers is a prerequisite for the excitation of vibrational HCN. Since the rotational transitions of $\mathrm{HCN}$ occur in the $\mathrm{mm}$ and sub-mm regimes the line emission can better penetrate the optically thick layers of dust, so it functions as an unattenuated tracer of deeply buried high mid-infrared surface brightness nuclei. 


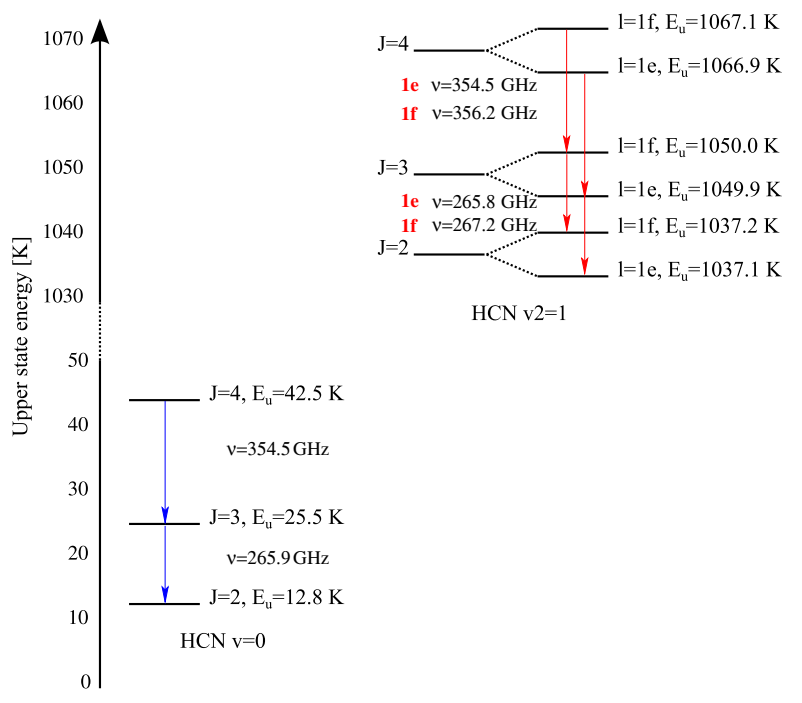

Fig. 1. HCN energy diagram with the HCN $v=0$ and $v_{2}=1 J=4$, 3,2 levels marked. The split of the $v_{2}=1$ rotational levels into $e$ and $f$ is also shown. The energy levels are not to scale which is illustrated by the dashed $y$-axis. The transitions are indicated with arrows. The values for the energy levels are from the Cologne Database for Molecule Spectroscopy (CDMS) ${ }^{1}$.

Extragalactic vibrationally excited $\mathrm{HCN}$ has been detected in the LIRG NGC 4418 (Sakamoto et al. 2010) and the ULIRGs Arp220 (Salter et al. 2008), IRAS 20551-4250 (Imanishi \& Nakanishi 2013), and Mrk231 (Aalto et al. 2015). In NGC 4418 and Arp220, vibrational lines of $\mathrm{HC}_{3} \mathrm{~N}$ have also been used to study the properties of the buried nucleus (Costagliola \& Aalto 2010; Costagliola et al. 2013, 2015; Sakamoto et al. 2010; Martín et al. 2011; Sakamoto et al. 2013). Vibrational temperatures ranging from 200 to $400 \mathrm{~K}$ have been estimated for $\mathrm{HCN}$ and $\mathrm{HC}_{3} \mathrm{~N}$ in NGC 4418, Arp220 and Mrk231. Vibrationally excited $\mathrm{HCN}$ has been detected in the circum-nuclear disk at the centre of the Milky Way by Mills et al. (2013), but line luminosities are significantly lower (relative to those of $v=0 \mathrm{HCN}$ ) compared to those in the galaxies studied here.

In this paper we present IRAM Plateau de Bure A-array observations of $J=3 \rightarrow 2, v=0 \mathrm{HCN}, \mathrm{HCO}^{+}$and $v_{2}=1 \mathrm{HCN}$ emission in the LIRGs IC 860 and Zw049.057. We also present Atacama Large Millimeter Array (ALMA) observations of the corresponding $J=4 \rightarrow 3$ lines in the ULIRGs Arp220 and IRAS 17208-0014. These new observations double the number of galaxies with detected vibrationally excited HCN emission and reveal in unprecedented detail emission emerging from highly compact structures behind a temperature gradient. We can show, for the first time, that the ground vibrational state $\mathrm{HCN}$ and $\mathrm{HCO}^{+}$line emission becomes self- and continuum absorbed and fails to trace the inner, nuclear regions of these galaxies.

\section{Vibrationally excited $\mathrm{HCN}$}

The HCN molecule has degenerate bending modes in the IR and may absorb IR-photons to its first vibrational state (Fig. 1). The first excited bending state of HCN is doubly degenerate, so that each rotational level $v_{2}=1 J$ is split into a closely spaced pair of levels $\left(f\right.$ and $e$ ). The $J=3-2$ and 4-3 $v_{2}=1$ lines are rotational transitions within the vibrationally excited state that occur near the frequency of the corresponding $v=0$ lines. For

\footnotetext{
1 https://www.astro.uni-koeln.de/cdms
}

example, the $v_{2}=1 e$ 3-2 line is shifted by only $20 \mathrm{MHz}$ from the $v=0 \mathrm{HCN} 3-2$ line and therefore appears close to the centre of the HCN line for extragalactic sources while the $v_{2}=1 f$ line has rest frequency $v=267.1993 \mathrm{GHz}$, shifted by $358 \mathrm{MHz}$ $\left(+400 \mathrm{~km} \mathrm{~s}^{-1}\right)$ from the $\mathrm{HCO}^{+} 3-2$ line. The $v_{2}=1 e$ and $1 f$ line emission always have similar intensity (except for cases of population inversion).

We will hereafter refer to the $\mathrm{HCN} v=0$ lines as "HCN" and the vibrationally excited $\mathrm{HCN} v_{2}=1 f$ lines as "HCN-VIB".

\subsection{Mid-IR excitation of the HCN-VIB lines}

The energy above ground of the $v_{2}=1$ state starts at $T_{E / k}=$ $1024 \mathrm{~K}$ and the critical density of the HCN-VIB lines is large enough $\left(>10^{10} \mathrm{~cm}^{-3}\right)$ that collisional excitation is unlikely (Ziurys \& Turner 1986; Mills et al. 2013). Instead, the vibrational ladder can be excited through the absorption of mid-IR $14 \mu \mathrm{m}$ continuum emission. There is no sharp threshold, but at mid-IR background temperatures of $T_{\mathrm{B}}(14 \mu \mathrm{m}) \approx 100 \mathrm{~K}$ the pumping is efficient enough to start to excite the line (Carroll \& Goldsmith 1981; Aalto et al. 2007).

For a thorough investigation of the vibrationally excited HCN a multi-line, non-LTE analysis is required. For example, if the buried source is very hot, compact and luminous it may be necessary to also consider higher vibrational modes $\left(v_{1}\right.$ at $3.02 \mu \mathrm{m}$ and $v_{3}$ at $4.77 \mu \mathrm{m}$ ) for the excitation of HCN-VIB (Cernicharo et al. 2011). In a forthcoming paper we will present non-LTE models of HCN-VIB emission excited by buried IR sources (González-Alfonso et al., in prep.).

However, for the purpose of this discussion we will assume that HCN-VIB is excited primarly by $14 \mu \mathrm{m}$ continuum with a brightness temperature $T_{\mathrm{B}} \gtrsim 100 \mathrm{~K}$.

\section{Observations}

\subsection{Sample galaxies}

The studied galaxies (see Table 1) are part of a sample where we find evidence of compact nuclear activity with faint $158 \mu \mathrm{m}$ [C II] line emission relative to FIR continuum (Malhotra et al. 1997; Díaz-Santos et al. 2013) and/or that show prominent $14 \mu \mathrm{m}$ HCN absorption (Lahuis et al. 2007). The [C II] deficit correlates with degree of compactness of the IR emitting source and the $L_{\mathrm{FIR}} / M_{\mathrm{H}_{2}}$ (Díaz-Santos et al. 2013).

The studied galaxies also have unusually bright $\mathrm{HC}_{3} \mathrm{~N}$ line emission which we previously have suggested is due to a compact buried hot nucleus (Aalto et al. 2007; Costagliola \& Aalto 2010; Costagliola et al. 2011). Note that we only include the western nucleus of the ULIRG merger Arp220 in this study (hereafter Arp220W) which is the brighter of the two nuclei. The eastern nucleus is discussed in Martín et al. (in prep.)

\subsection{IRAM Plateau de Bure}

The $1 \mathrm{~mm}$ observations were carried out with the 6-element configuration in March 2014 in A-array. The receivers were tuned to a frequency of $263.0 \mathrm{GHz}$, to include both the redshifted HCN and $\mathrm{HCO}^{+} J=3-2$ lines $(v=265.886 \mathrm{GHz}$ and $267.558 \mathrm{GHz}$ rest frequency, respectively). We used the WideX correlator providing a broad frequency range of $3.6 \mathrm{GHz}$ and $2 \mathrm{MHz}$ spectral resolution. After calibration within the GILDAS reduction package, the visibility set was converted into FITS format, and imported into the AIPS package for further imaging. We adopted natural weighting during the CLEAN deconvolution. Table 1 
Table 1. Sample galaxies.

\begin{tabular}{|c|c|c|c|c|c|c|c|c|}
\hline Name & $\begin{array}{l}\text { RA } \\
(\mathrm{J} 2000)\end{array}$ & $\begin{array}{l}\text { Dec } \\
(\mathrm{J} 2000)\end{array}$ & $\begin{array}{c}\log L_{\mathrm{FIR}} \\
{\left[L_{\odot}\right]}\end{array}$ & $\begin{array}{c}D \\
{[\mathrm{Mpc}]}\end{array}$ & Type & Telescope & $\begin{array}{c}\text { Beam } \\
\left({ }^{\prime \prime}\right)\end{array}$ & $\begin{array}{l}\text { Sensitivity } \\
\text { (mJy/channel) }\end{array}$ \\
\hline IC 860 & $13: 15: 03.05$ & $+24: 37: 08.0$ & 11.17 & 59 & Sab & IRAM PdBI & $0 ! 50 \times 0 . ' 23$ & $1.2 \mathrm{mJy}$ \\
\hline Zw049.057 & $15: 13: 13.10$ & $+07: 13: 32.0$ & 11.27 & 59 & $\mathrm{Sab}$ & IRAM PdBI & $0.55 \times 0 . \prime 24$ & $1.6 \mathrm{mJy}$ \\
\hline Arp220W & $15: 34: 57.27$ & $+23: 30: 10.5$ & 12.03 & 80 & merger & ALMA & $0 . ' 44 \times 0 . \prime 38$ & $1.9 \mathrm{mJy}$ \\
\hline IRAS $17208-0014$ & $17: 23: 21.94$ & $-00: 17: 01.0$ & 12.39 & 176 & merger & ALMA & $0 ! 49 \times 0.34$ & $1.6 \mathrm{mJy}$ \\
\hline
\end{tabular}

Notes. $L_{\mathrm{IR}}$ and $D$ from Sanders et al. (2003) (where $D$ is the angular distance $\left(D_{\mathrm{A}}\right)$ ) but for Arp220W we attempt to estimate the luminosity emerging from the western nucleus only from the continuum study of Sakamoto et al. (2008). ${ }^{(\star)}$ For channel widths of $33 \mathrm{~km} \mathrm{~s}^{-1}$ (IC 860 and Zw049.057) and $20 \mathrm{~km} \mathrm{~s}^{-1}$ (Arp220W and IRAS 17208-0014).

gives the beam sizes and sensitivities of the final data. The details of the observations are:

IC 860: the bandpass of the individual antennas was derived from the bright quasar 3C84 and the absolute flux calibration was set on MWC349. The quasars J1310+323 ( 0.7 Jy at $1 \mathrm{~mm})$ and $\mathrm{J} 1328+307(\sim 0.2 \mathrm{Jy})$ were observed regularly for complex gain calibration every $25 \mathrm{~min}$.

Zw049.057: the bandpass and flux calibrations were derived from 3C 279 and MWC349, respectively. The gain calibration was derived from observations of J1502+106 ( $0.4 \mathrm{Jy})$ and $\mathrm{J} 1456+044(\sim 0.2 \mathrm{Jy})$ with a similar scheme as for IC 860 .

\subsection{ALMA}

The final sensitivity and beam sizes of the observations are given in Table 1 and the details are discussed below.

IRAS 17208-0014: the $0.8 \mathrm{~mm}$ observations were carried out with ALMA (with 34 antennas in the array) in May 2014. The correlator was set up to cover two contiguous bands of $1.875 \mathrm{GHz}$ in each of the lower (centered at $\sim 341.9 \mathrm{GHz}$ ) and upper (centered at $\sim 352.1 \mathrm{GHz}$ ) sideband, with 960 spectral channels (1.95 MHz wide each) per band. The upper side band includes the $\operatorname{HCN} J=4-3 v=0$ and $v=1 f$ lines. The bandpass of the individual antennas was derived from the bright quasar J1733-1304. The quasar J1751+096 ( 1.7 Jy) was observed regularly for complex gain calibration. Its flux was extracted from the ALMA flux-calibrator database for absolute flux calibration. After calibration within the CASA reduction package, the visibility set was imported into the AIPS package for further imaging.

Arp220: the $0.8 \mathrm{~mm}$ observations were carried out with ALMA in April and May 2014 (with 34 and 30 usable antennas, respectively). Spectral features presented here were observed in the USB with $2 \times 1.875 \mathrm{GHz}$ spectral windows centered in 347.8 and $349.6 \mathrm{GHz}$. The bandpass of the individual antennas was derived from the quasar $\mathrm{J} 1550+0527$. The flux calibration was set on Titan, where intrinsic atmospheric lines were masked. The quasar J1516+1932 ( 0.4 Jy at $0.9 \mathrm{~mm})$ was observed regularly for complex gain calibration every 5 min. Calibration, deconvolution and imaging were carried out in the CASA reduction package.

\section{Results}

Line fluxes, luminosities and source sizes are presented in Table 2, nuclear (central beam) spectra in Figs. 2 and 3.

\section{1. $\mathrm{HCN}$ and $\mathrm{HCO}^{+}$}

$\mathrm{HCN}$ and $\mathrm{HCO}^{+}$source diameters (FWHM) range between 100 and $400 \mathrm{pc}$ (Table 2) and the line profiles have a striking double-peaked shape with a minimum near systemic velocity (see Figs. 2 and 3).

\subsubsection{Line shapes}

In Sect. 5.1 we present strong evidence that the double-peaked $\mathrm{HCN}$ and $\mathrm{HCO}^{+}$spectra are caused by deep self- and continuum absorption at systemic velocity. Therefore, FWHM widths cannot be fitted for these lines. In IC 860, the shapes of the HCN and $\mathrm{HCO}^{+}$spectra appear as reversed $P$-Cygni profiles - strongly asymmetric with the red part of the line profile showing stronger absorption. This is particularly striking for $\mathrm{HCO}^{+}$where the continuum is also absorbed on the red side. The deepest absorption feature occurs at a projected velocity of $70 \mathrm{~km} \mathrm{~s}^{-1}$. The emission peak observed between the $\mathrm{HCN}-\mathrm{VIB}$ line and the $\mathrm{HCO}^{+}$ absorption feature is part of the unabsorbed high velocity $\mathrm{HCO}^{+}$ (similar to what is seen in $\mathrm{HCN}$ where the absorption is less pronounced).

In the other galaxies the absorption profiles are instead somewhat blue-shifted by -50 to $-100 \mathrm{~km} \mathrm{~s}^{-1}$ for Zw049.057 and -100 to $-300 \mathrm{~km} \mathrm{~s}^{-1}$ for Arp220W and IRAS 17208-0014.

\subsection{Vibrationally excited $\mathrm{HCN}$}

We find luminous HCN-VIB $J=3-2$ or 4-3 $1 f$ line emission in all four sample galaxies. The emission originates from the very nucleus of the galaxies with upper limit diameters (FWHM) ranging from 37 to $136 \mathrm{pc}$ (Table 2). For the ULIRG merger IRAS 17208-0014 the HCN-VIB emission appears to be associated primarily with the western of the two nuclei found by Medling et al. (2014). For all four galaxies, the HCN-VIB emission is significantly more compact (by factors of at least 2-3) than that of $\mathrm{HCN}$ and $\mathrm{HCO}^{+}$and for IC 860 the integrated strength of the line exceeds that of $\mathrm{HCO}^{+}$in the nucleus.

The HCN-VIB line widths range from 130 to $450 \mathrm{~km} \mathrm{~s}^{-1}$ and the emission peaks close to systemic velocity. This is most clearly seen in the two LIRGs where the line blending with $\mathrm{HCO}^{+}$is less significant. The centre (systemic) velocities are marked with a dashed line in Figs. 2 and 3 and for IC 860 it is estimated to $c z=3880 \pm 20 \mathrm{~km} \mathrm{~s}^{-1}$ from the position velocity $(\mathrm{pV})$ diagram. The centre velocity for Zw049.057 is very similar $c z=3900 \pm 20 \mathrm{~km} \mathrm{~s}^{-1}$ where the errors also stem from the $\mathrm{pV}$ diagram (see Sect. 4.4). For Arp220W the line marks $c z=5434 \mathrm{~km} \mathrm{~s}^{-1}$ which is $17 \mathrm{~km} \mathrm{~s}^{-1}$ below the systemic velocity estimated for Arp220W by Sakamoto et al. (2008). It is suggested that the systemic velocity of IRAS 17208-0014 is $c z=12834 \mathrm{~km} \mathrm{~s}^{-1}$ (Downes et al. 1993) (also marked with a 

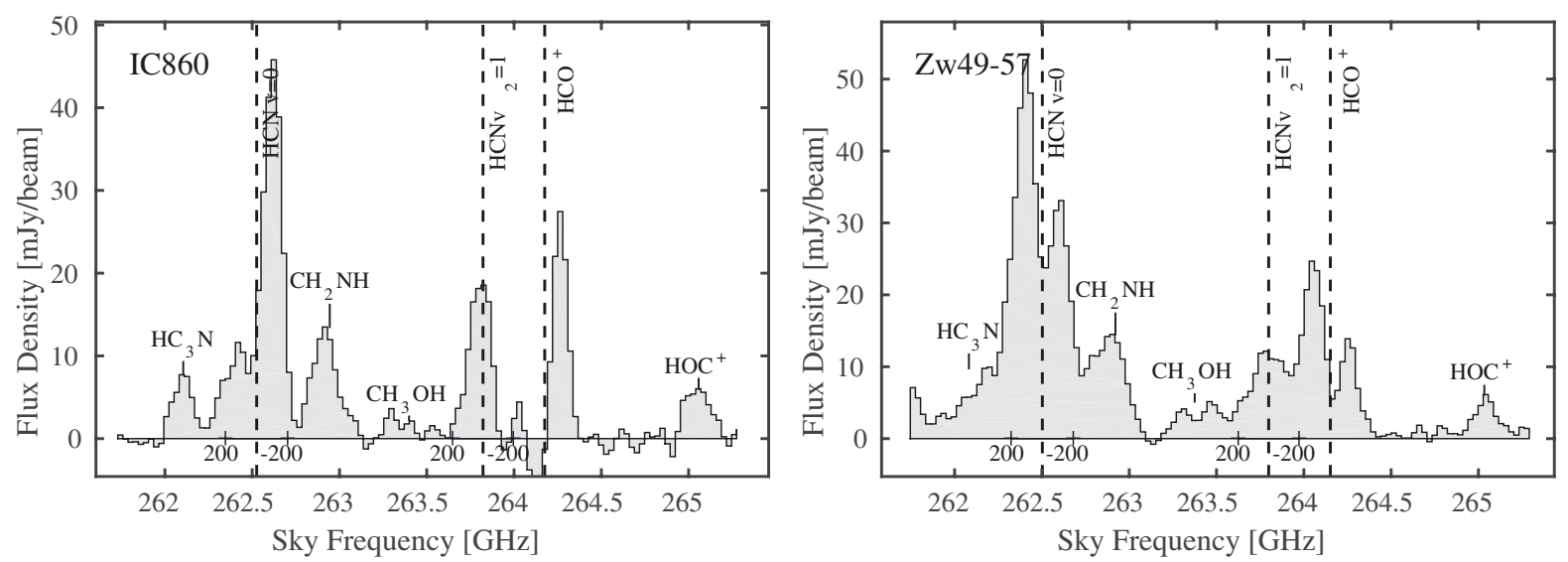

Fig. 2. IRAM Plateau de Bure spectra (continuum subtracted) of IC 860 (left panel) and Zw049.057 (right panel). The systemic velocities of $\mathrm{HCN}, \mathrm{HCO}^{+}$and $\mathrm{HCN}-\mathrm{VIB} J=3-2$ are marked with dashed vertical lines. The self- and continuum absorption leads to double-peaked HCN and $\mathrm{HCO}^{+} v=0$ spectra while the HCN-VIB lines are singled peaked close to systemic velocity (see Sect. 4.2). The spectra also show prominent, single peaked lines of $\mathrm{HOC}^{+}, \mathrm{CH}_{2} \mathrm{NH}, \mathrm{CH}_{3} \mathrm{OH}$ and $\mathrm{HC}_{3} \mathrm{~N}$. Fluxes are in mJy beam ${ }^{-1}$.
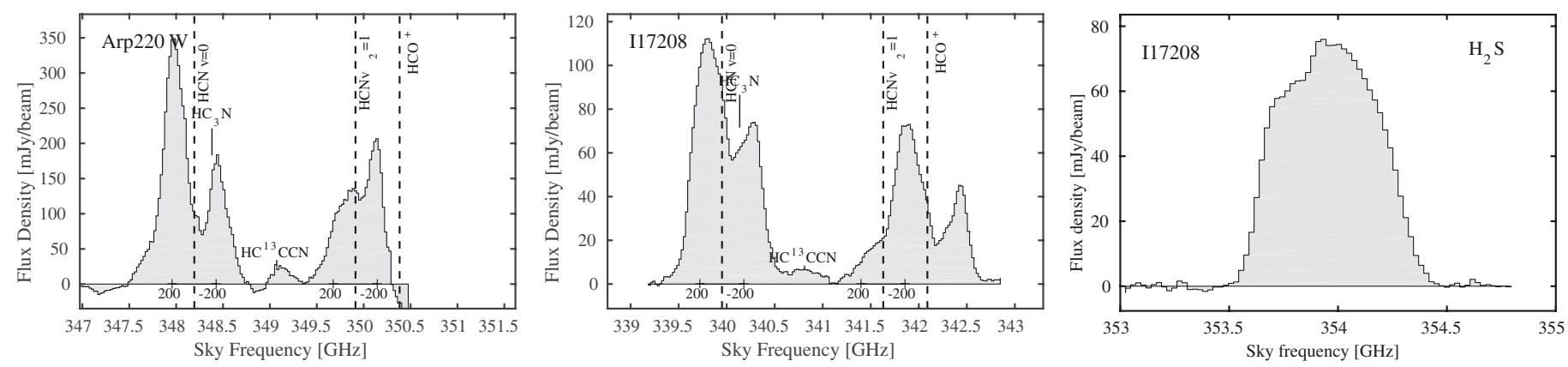

Fig. 3. ALMA spectra (continuum subtracted) of Arp220W (left panel) and IRAS 17208-0014 (centre and right panels). The systemic velocities of $\mathrm{HCN}, \mathrm{HCO}^{+}$and $\mathrm{HCN}-\mathrm{VIB} J=4-3$ and the $\mathrm{H}_{2} \mathrm{~S}$ lines are marked with dashed vertical lines. The self- and continuum absorption leads to double-peaked $\mathrm{HCN}$ and $\mathrm{HCO}^{+} v=0$ spectra while the $\mathrm{HCN}-\mathrm{VIB}$ and $\mathrm{H}_{2} \mathrm{~S}$ lines are single-peaked close to systemic velocity (see Sect. 4.2). Half of the $\mathrm{HCN}-\mathrm{VIB}$ is blended with the $\mathrm{HCO}^{+}$line. Note that part of the $\mathrm{HCO}^{+}$line is outside the band for Arp220W. We also detect lines of $\mathrm{HC}_{3} \mathrm{~N}$ and $\mathrm{HC}^{13} \mathrm{CCN}$. Fluxes are in mJy beam ${ }^{-1}$.

dashed line in Fig. 3) while Garcia-Burillo et al. (2015) suggest that the systemic velocity is blue-shifted by $30 \mathrm{~km} \mathrm{~s}^{-1}$ from this value. We have indicated this lower value of the centre velocity in the pV diagram for IRAS 17208-0014 (Fig. 5).

\subsection{Other species}

For IC 860 and Zw049.057 (see Fig. 2) we detect the $\mathrm{HC}_{3} \mathrm{~N}$ $J=29-28 v_{7}=2$ line $\left(T_{E_{1} / k}=819 \mathrm{~K}\right)$ on the redshifted and the $\left(4_{1,3}-3_{1,2}\right)$ transition of $\mathrm{CH}_{2} \mathrm{NH}$ at $266 \mathrm{GHz}$ (with energy level $T_{E_{1} / k}=27 \mathrm{~K}$ ) on the blue side of the HCN 3-2 line and several blended $\mathrm{CH}_{3} \mathrm{OH}$ lines between $\mathrm{CH}_{2} \mathrm{NH}$ and HCN-VIB. It is interesting to note that Salter et al. (2008) find bright lines of $\mathrm{CH}_{2} \mathrm{NH}$ in their cm-wave spectral scan of Arp220. We also detect very bright $\mathrm{HOC}^{+} 3-2$ line emission on the blue side of the $\mathrm{HCO}^{+} 3-2$ line.

For Arp220W and IRAS 17208-0014 (see Fig. 3) bright $J=$ $39-38 \mathrm{HC}_{3} \mathrm{~N}$ line emission is blended with the HCN 4-3 line and $\mathrm{H}^{13} \mathrm{CCCN}$ is found in between the HCN and HCN-VIB line. For IRAS 17208-0014 we have also detected luminous line emission from the $3_{2,1}-3_{1,2}$ and $4_{3,1}-4_{2,2}$ transitions of ortho- $\mathrm{H}_{2} \mathrm{~S}$ and para- $\mathrm{H}_{2} \mathrm{~S}$ at $369 \mathrm{GHz}$. The two lines are shifted only $26 \mathrm{MHz}$ from each other (energy levels $T_{E_{1} / k}=137 \mathrm{~K}$ and $245 \mathrm{~K}$ respectively) so they are effectively blended into one line.

\subsection{Position velocity diagrams}

For IC 860 and Zw049.057 the HCN-VIB emission is spatially unresolved and peaks at velocities close to systemic (Fig. 4). In comparison, the brightest $\mathrm{HCN}$ emission occurs outside the HCN-VIB at higher absolute velocities, with a velocity difference between the two peaks of $230-260 \mathrm{~km} \mathrm{~s}^{-1}$. Even though much less pronounced than for the $\mathrm{HCN}$ and $\mathrm{HCO}^{+}$lines, the HCN-VIB line also seems to be slightly double-peaked. The velocity shift between the peaks is relatively small: $\approx 80 \mathrm{~km} \mathrm{~s}^{-1}$.

For the ULIRGs Arp220W and IRAS 17208-0014 the HCNVIB line is broad enough for it to be partially blended with the $\mathrm{HCO}^{+}$line (see example for IRAS 17208-0014 in Fig. 5). Observations at higher spatial resolution are necessary to completely disentangle the HCN-VIB emission. The HCNVIB pV diagrams for both Arp220W (to be presented in Martín et al., in prep.) and IRAS 17208-0014 (Fig. 5) therefore only show half of the line but in both cases a single peak at systemic velocity is suggested. For IRAS $17208-0014$ the $369 \mathrm{GHz}$ $\mathrm{H}_{2} \mathrm{~S}$ line has a profile that does not show the pronounced double structure of $\mathrm{HCN}$ and $\mathrm{HCO}^{+}$. The $\mathrm{pV}$ diagram (Fig. 5) reveals that the $\mathrm{H}_{2} \mathrm{~S}$ line emission peaks where $\mathrm{HCN}$ and $\mathrm{HCO}^{+}$have a minimum. Note also the blue-shifted high-velocity extension to the HCN line which may be the HCN counterpart to the CO 2-1 high velocity outflow detected by Garcia-Burillo et al. (2015). 
S. Aalto et al.: Probing highly obscured, self-absorbed galaxy nuclei with vibrationally excited HCN
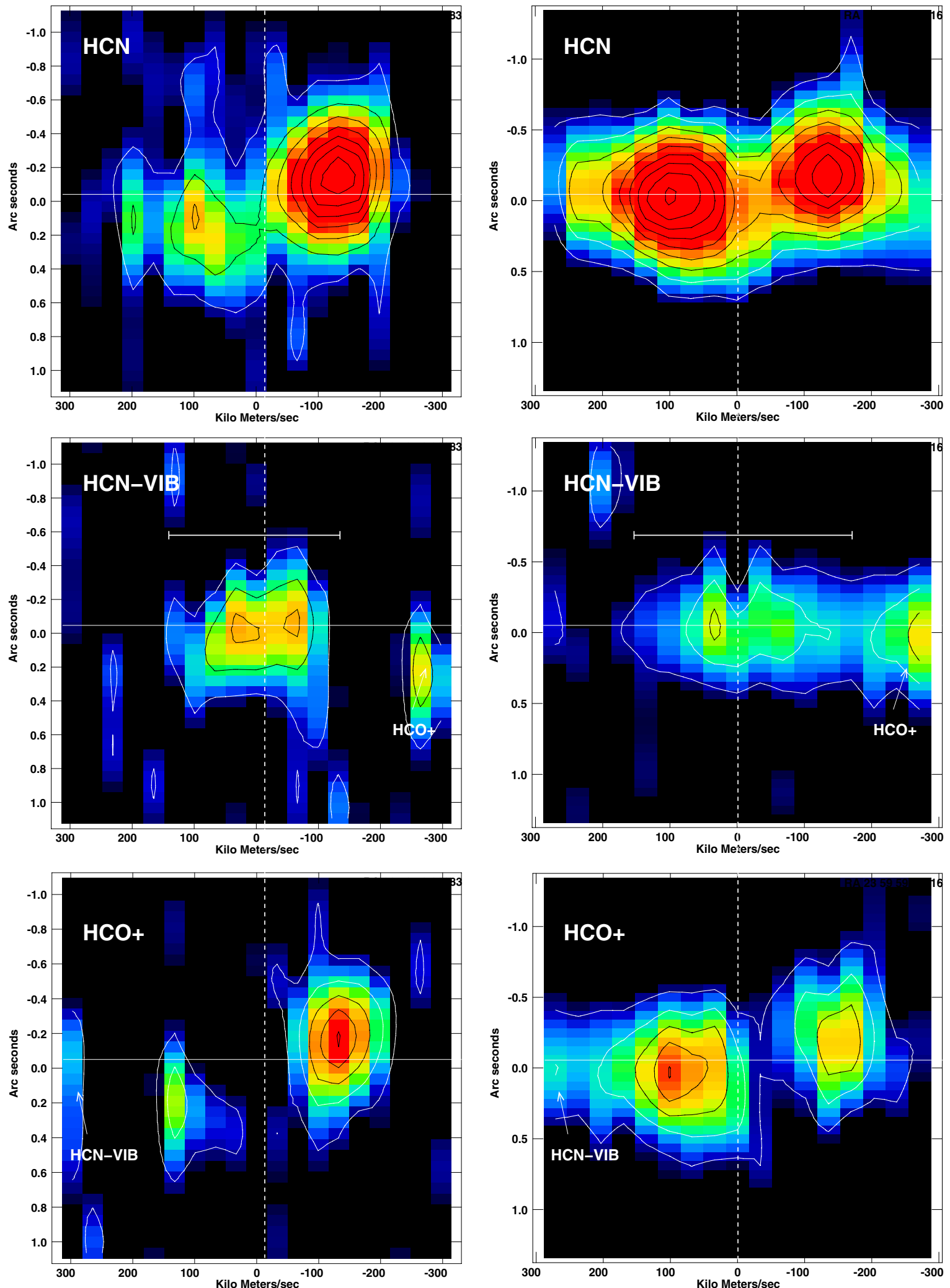

Fig. 4. Position velocity (pV) diagrams (continuum subtracted) for IC 860 (left) and Zw049.057 (right) where the $x$-axis shows the radial velocity (relative to $c z=3880 \mathrm{~km} \mathrm{~s}^{-1}$ and $3900 \mathrm{~km} \mathrm{~s}^{-1}$ respectively) and the $y$-axis shows the position along the major axis (for both galaxies the position angle is $-20^{\circ}$ ). The upper panel shows the $\mathrm{HCN}$, the centre the $\mathrm{HCN}-\mathrm{VIB}$ and the lower panel the $\mathrm{HCO}^{+} \mathrm{pV}$ diagrams. The contours are $3.5 \times$ $(1,3,5,7,9,11,13) \mathrm{mJy}_{\text {beam }}^{-1}$ (IC 860) and $3.5 \times\left(1,3,5, \ldots\right.$ 17) $\mathrm{mJy} \mathrm{beam}^{-1}$ (for Zw049.057). The colour ranges from 2 to $30 \mathrm{mJy}^{\mathrm{beam}}{ }^{-1}$. The white vertical dashed line indicate the systemic velocity. The horizontal white line indicates the nuclear position of the HCN-VIB line. 

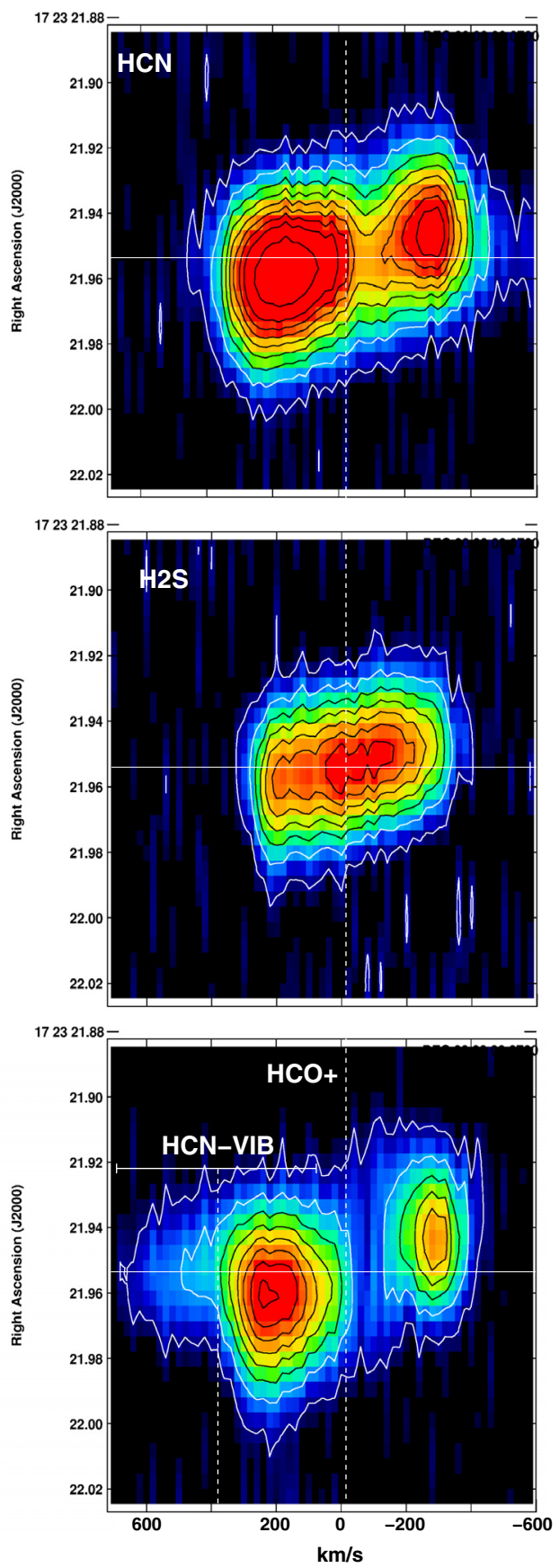

Fig. 5. Position velocity (pV) diagrams (continuum subtracted) for IRAS 17208-0014 where the $y$-axis shows the position along the major axis (position angle $90^{\circ}$ ) and the $x$-axis shows the radial velocity relative to the $c z$ of $17208-0014 \mathrm{~km} \mathrm{~s}^{-1}$. Upper panel: HCN 4-3. Centre panel: $\mathrm{H}_{2} \mathrm{~S}$ Lower panel: $\mathrm{HCO}^{+} 4-3$ blended with $\mathrm{HCN}-\mathrm{VIB}$. Colour ranges from 2 to $70 \mathrm{mJy}$, contour levels are $5 \times(1,3,5, \ldots 17) \mathrm{mJy}^{-1}$ beam $^{-1}$ The vertical dashed line marks $z=0.04271$ the suggested redshift by Garcia-Burillo et al. (2015), while zero velocity marks the redshift $z=0.04281$. The horizontal white bar indicates the nuclear position of the HCN-VIB line.

It may be similar to the HCN 3-2 outflow already found in the ULIRG Mrk231 by Aalto et al. (2015).

\subsection{Continuum}

Continuum fluxes and FWHM source sizes at 260 or $350 \mathrm{GHz}$ can be found in Table 3. The FWHM size of the continuum emission region is larger than the HCN-VIB emission for two sources - Zw049.057 and IRAS 17208-0014. In IC 860 the continuum source is unresolved as is the HCN-VIB emission. Sakamoto et al. (2008) and Wilson et al. (2014) have shown the existence of an optically thick and compact submm continuum source in Arp220W consistent with our result. In both IRAS 17208-0014 and IC 860 the lower limit to the brightness temperature of the 260 and $350 \mathrm{GHz}$ continuum sources is $T_{\mathrm{B}}>40 \mathrm{~K}$ suggesting that these sources may also be optically thick.

\section{Discussion}

\subsection{Self-absorption in the $\mathrm{HCN}$ and $\mathrm{HCO}^{+}$lines}

The nuclear $\mathrm{HCN}$ and $\mathrm{HCO}^{+}$spectra are double peaked in contrast to the HCN-VIB lines (and also the $\mathrm{CH}_{2} \mathrm{NH}, \mathrm{H}_{2} \mathrm{~S}$ and $\mathrm{HOC}^{+}$lines). We argue that this is due to a combination of selfand continuum absorption in the $\mathrm{HCN}$ and $\mathrm{HCO}^{+}$lines where photons at the line centre are reabsorbed by cooler gas in the external parts of the HCN-VIB emitting core and plausibly also in more extended regions. (see Fig. 6 for an illustration). A temperature gradient and high column densities will therefore result in self-absorbed lines as seen also in embedded sources in the Milky Way (Rolffs et al. 2011b). The self-absorption obliterates any sign of the hot dense nucleus in the $\mathrm{HCN}$ and $\mathrm{HCO}^{+}$ lines impeding their use as tracers of the properties of extremely dust-embedded nuclei. The impact of self-absorption is further discussed in Sect. 5.2 below.

How do we know that the twin-peaked $\mathrm{HCN}$ and $\mathrm{HCO}^{+}$lines are not caused by a real absence of dense molecular gas in the nucleus? We need other lines to compare with to determine the cause of the absorption.

\subsubsection{Evidence from the $\mathrm{HCN}-\mathrm{VIB}$ line}

We can test the self-absorption theory for $\mathrm{HCN}$ through comparing the intensity in the vibrational $v_{2}$ state (both the $1 e$ and $1 f$ line) to that of the vibrational ground state $-I_{\mathrm{u}} / I_{1}$ in Eq. (B.1)). Unless the HCN-VIB line is masing, $I_{\mathrm{u}}<I_{1}$. The $I_{\mathrm{u}}=I(\mathrm{HCN}-\mathrm{VIB})(1 e+1 f)$ and for thermal excitation the $1 e$ line is as bright as the $1 f$ line and hence $I_{\mathrm{u}}=2 \times I(\mathrm{HCN}-\mathrm{VIB})$. Thus, for $I_{\mathrm{u}}<I_{1}$ to hold, the $H C N$-VIB/HCN line intensity ratio, $\mathcal{R}_{\mathrm{vib}}$, should be $<0.5$. Note, that here we are assuming that all of the $\mathrm{HCN}$ emission is emerging from the same gas that produces the HCN-VIB line. Any additional HCN-emission would further lower the "allowed" $\mathcal{R}_{\text {vib }}$ ratio.

We can now inspect the nuclear spectra and determine $\mathcal{R}_{\text {vib }}$ on a channel-by-channel basis. For IC 860 and Arp220W $\mathcal{R}_{\text {vib }}$ exceeds unity close to systemic velocities and therefore line photons of both ground state $\mathrm{HCN}$ and $1 e \mathrm{HCN}-\mathrm{VIB}$, which appears close to the HCN $v=0$ line centre (see Sect. 2), are being absorbed (see zoomed-in example for IC 860 in Fig. 7). However for $Z w 049.057$ the ratio is $\mathcal{R}_{\text {vib }}=0.5$ and for IRAS 17208$0014 \mathcal{R}_{\mathrm{vib}}=0.25$ and additional evidence is required to claim that the $\mathrm{HCN}$ and $\mathrm{HCO}^{+}$line shapes are due to self-absorption. Furthermore, continuum absorption by the optically thick $\mathrm{HCN}$ and $\mathrm{HCO}^{+}$lines can be important, given that the continuum brightness is similar to the continuum-subtracted line brightness at central velocities.

\subsubsection{Comparing with other lines}

We can use other, optically thin, species to trace the dense gas in the nuclear region. In Appendix $\mathrm{A}$ we discuss the $\mathrm{CH}_{2} \mathrm{NH}$ line emission in $\mathrm{Zw} 049.057$ and the $\mathrm{H}_{2} \mathrm{~S}$ line emission of I17208-0014 and how they support the notion of self-absorbed 
Table 2. Line flux densities.

\begin{tabular}{|c|c|c|c|c|c|c|}
\hline Line & $\begin{array}{c}\text { Peak }^{\dagger} \\
{\left[\mathrm{Jy} \mathrm{km} \mathrm{s}^{-1} \text { beam }^{-1}\right]}\end{array}$ & $\begin{array}{l}\text { Integrated } \\
{\left[\mathrm{Jy} \mathrm{km} \mathrm{s}^{-1}\right]}\end{array}$ & $\begin{array}{c}L \\
{\left[L_{\odot} \times 10^{3}\right]}\end{array}$ & $\begin{array}{l}\theta^{(\dagger)} \\
{[\mathrm{pc}]}\end{array}$ & $\begin{array}{l}\mathrm{PA}^{\dagger} \\
{\left[{ }^{\circ}\right]}\end{array}$ & $\begin{array}{c}\Delta \mathrm{V} \\
{\left[\mathrm{km} \mathrm{s}^{-1}\right]}\end{array}$ \\
\hline \multicolumn{7}{|l|}{ IC 860} \\
\hline HCN 3-2 & $8.8 \pm 0.3$ & $19.4 \pm 0.7$ & 18.5 & $128 \times 80$ & 20 & \\
\hline HCN-VIB 3-2 & $3.8 \pm 0.4$ & $4.0 \pm 0.4$ & 3.8 & $<37$ & & 130 \\
\hline $\mathrm{HCO}^{+} 3-2$ & $3.3 \pm 0.2$ & $9.0 \pm 0.4$ & 8.6 & $133 \times 94$ & 20 & $\ldots$ \\
\hline $\mathrm{CH}_{2} \mathrm{NH}$ & $2.2 \pm 0.2$ & $3.4 \pm 0.4$ & 3.2 & $<69$ & $\ldots$ & 160 \\
\hline $\mathrm{HOC}^{+}$ & $1.1 \pm 0.2$ & $1.3 \pm 0.3$ & 1.2 & $<0.2$ & $\ldots$ & 140 \\
\hline \multicolumn{7}{|l|}{ Zw049.057 } \\
\hline HCN 3-2 & $18.8 \pm 0.4$ & $64.4 \pm 1.5$ & 60.7 & $172 \times 137$ & 25 & \\
\hline HCN-VIB 3-2 & $4.2 \pm 0.2$ & $6.8 \pm 0.5$ & 6.4 & $<86$ & $\ldots$ & 250 \\
\hline $\mathrm{HCO}^{+} 3-2$ & $7.3 \pm 0.2$ & $25.3 \pm 0.7$ & 23.8 & $133 \times 94$ & 30 & \\
\hline $\mathrm{CH}_{2} \mathrm{NH}$ & $4.6 \pm 0.3$ & $11.3 \pm 1.1$ & 10.8 & $154 \times 80$ & $\ldots$ & 250 \\
\hline $\mathrm{HOC}^{+}$ & $1.7 \pm 0.2$ & $2.6 \pm 0.4$ & 2.5 & $<0.4$ & $\ldots$ & 200 \\
\hline \multicolumn{7}{|l|}{ Arp220W } \\
\hline $\mathrm{HCN} 4-3$ & $130 \pm 5$ & $294 \pm 15$ & 670 & $310 \times 250$ & 146 & \\
\hline HCN-VIB 4-3 & $55 \pm 3$ & $70 \pm 5$ & 160 & $62 \times 50$ & 133 & $350^{\star}$ \\
\hline $\mathrm{HCO}^{+} 4-3$ & $55 \pm 2$ & $123 \pm 7$ & 286 & $310 \times 250$ & 140 & $\ldots$ \\
\hline \multicolumn{7}{|l|}{ IRAS 17208} \\
\hline $\mathrm{HCN} 4-3$ & $53.7 \pm 0.1$ & $86.0 \pm 0.2$ & 941 & $307 \times 256$ & 95 & $\ldots$ \\
\hline HCN-VIB 4-3 & $8.0 \pm 0.2$ & $8.0 \pm 0.2$ & 87 & $<136$ & $\ldots$ & $450^{\star}$ \\
\hline $\mathrm{HCO}^{+} 4-3$ & $30.8 \pm 0.05$ & $55.8 \pm 0.05$ & 610 & $350 \times 299$ & 100 & \\
\hline $\mathrm{H}_{2} \mathrm{~S}$ & $36.3 \pm 0.1$ & $45.3 \pm 0.2$ & 496 & $196 \times 136$ & 95 & 450 \\
\hline
\end{tabular}

Notes. ${ }^{(\dagger)}$ Calculated from two-dimensional Gaussian fits to the integrated intensity emission using the AIPS task IMFIT which deconvolves the clean beam from the fitted component size. The accuracy and errors of this process are discussed in Condon (1997). $\left.{ }^{\star}\right)$ From Gaussian fits where the line is blended with $\mathrm{HCO}^{+}$. Limits to $\mathrm{HCN}-\mathrm{VIB}$ brightness temperatures are $T_{\mathrm{B}}$ (HCN-VIB) $>20 \mathrm{~K}$ for IC 860 , $>6 \mathrm{~K}$ for Zw049.057, $>8 \mathrm{~K}$ for IRAS $17208-0014$ and $68 \mathrm{~K}$ for Arp220W. For IC 860 and Zw049.057 $1^{\prime \prime}=286 \mathrm{pc}$, for Arp220W $1^{\prime \prime}=388$ pc and for IRAS $17208-$ $00141^{\prime \prime}=853 \mathrm{pc}$.

Table 3. Continuum fluxes and source sizes.

\begin{tabular}{lcccc}
\hline \hline Name & $\begin{array}{c}\text { Peak } \\
{[\mathrm{mJy}]}\end{array}$ & $\begin{array}{c}\text { Integrated } \\
{[\mathrm{mJy}]}\end{array}$ & $\begin{array}{c}\theta \\
{[\mathrm{pc}]}\end{array}$ & $\begin{array}{c}T_{\mathrm{B}} \\
{[\mathrm{K}]}\end{array}$ \\
\hline IC 860 & $35 \pm 2$ & $35 \pm 2$ & $<37$ & $>43$ \\
Zw 049.057 & $20 \pm 1$ & $44 \pm 3$ & $145 \times 86$ & $>5$ \\
Arp220W & $283 \pm 3$ & $384 \pm 4$ & $62 \times 50$ & $>160$ \\
IRAS 17208 & $87 \pm 1$ & $116 \pm 2$ & $248 \times 162$ & $>40$ \\
\hline
\end{tabular}

Notes. Continuum levels were determined through a zeroth-order fit to line-free channels in the uv-plane. Source sizes (diameters) are full width half maximum (FWHM) two-dimensional Gaussian fits. The continuum fluxes and source sizes are determined for $260 \mathrm{GHz}$ for IC 860 and Zw049.057 and for $350 \mathrm{GHz}$ for IRAS 17208-0014 and Arp220. The continuum values for Arp220W are from Martín et al. (in prep.) and are consistent with those reported in Sakamoto et al. (2008).

$\mathrm{HCN}$ and $\mathrm{HCO}^{+}$in these galaxies. In Appendix A.2 we also discuss how the $\mathrm{HCO}^{+} / \mathrm{HOC}^{+}$ratio is also consistent with self-absorbed $\mathrm{HCO}^{+}$.

\subsection{Impact of self-absorbed $\mathrm{HCN}$ and $\mathrm{HCO}^{+}$lines in galaxy nuclei}

The effect of self absorption on the $\mathrm{HCN}$ and $\mathrm{HCO}^{+}$lines (see illustration in Fig. 6) demonstrates that the emission cannot be viewed as emerging from an ensemble of clouds that are independent of each other - but that emission from clouds closer to the nucleus is being absorbed by clouds farther out. The notion that the spectrum observed towards a galaxy involves "counting clouds" - that may be individually optically thick but collectively "optically thin" (i.e., not overlapping each other in the 2D (sky plane) or the velocity (line-of-sight) space) - does not hold for these galaxy nuclei.

This breaking down of the ensemble approximation has been predicted by Downes et al. (1993) who suggested that (U)LIRG nuclei should be viewed as a single cloud. The ensemble approximation is appropriate when the mean gas surface density is low or moderate, but becomes less and less valid when observing a dense ULIRG/compact nucleus at increasing resolution. A similar conclusion that emission is not emerging from individual, non-overlapping clouds in the nuclei of Arp220 was also drawn by Scoville et al. (2015).

When lines of $\mathrm{HCN}$ and $\mathrm{HCO}^{+}$become self-absorbed they cannot be reliably used to probe the central regions of galaxies on scales where the absorption occurs - for our sample galaxies this means $r<100 \mathrm{pc}$. For the studied galaxies this is also where most of the emission comes from, in contrast to many nearby galaxies with central molecular zones extended over larger regions. Thus, the absorbed $\mathrm{HCN}$ and $\mathrm{HCO}^{+}$line profiles are strongly impacting our understanding also of the global properties of these galaxies. Very recently self-absorbed CO 6-5 has been detected towards both nuclei of Arp220 by Rangwala et al. (2015).

\subsection{HCN-VIB lines as probes of extreme mid-IR surface brightness and luminosity}

Vibrationally excited lines of $\mathrm{HCN}$ are excellent probes of the nuclear dynamics and serve as proxies for the mid-IR luminosity density in deeply obscured nuclei where $N\left(\mathrm{H}_{2}\right)>10^{24}$ $\mathrm{cm}^{-2}$ and where dust temperatures exceed $100 \mathrm{~K}$. Mid-IR excitation of HCN is favoured in regions of high dust temperature and with significant gas and dust column densities. This is 
because an $T_{\mathrm{B}}(14 \mu \mathrm{m}) \gtrsim 100 \mathrm{~K}$ is necessary to start to excite the HCN vibrational ladder and this requires an optically thick mid-IR source - which in turn requires an $\mathrm{H}_{2}$ column density $N\left(\mathrm{H}_{2}\right) \gtrsim 2 \times 10^{23} \mathrm{~cm}^{-2}$ (see Appendix B for a discussion). Note that here we assume that the molecules receive the exciting radiation isotropically. If they do not then the background source must be even hotter to make up for the loss in solid angle.

The HCN-VIB lines of the sample galaxies are very luminous (see discussion in Sect. 5.5.1) and lower limits to $T_{\mathrm{B}}(\mathrm{HCN}-$ VIB) exceed $20 \mathrm{~K}$ for IC 860 and Arp220W (see footnote to Table 2). Therefore, the filling factor of hot dust and gas is extremely high in the inner tens of pc of the sample galaxies much higher than on similar size scales in Milky Way massive star forming regions or in extended starbursts. The implication is that the HCN-VIB emission we detect towards these galaxies cannot be emerging from a collection of Milky-Way-like hot cores. The high filling factor of hot, optically thick gas and dust suggests that we can use the minimum $14 \mu \mathrm{m}$ brightness temperature of $100 \mathrm{~K}$ to estimate a lower limit to the mid-infared luminosity.

We assume a nuclear spherical "cloud" of dust for which we adopt a simple blackbody where $L=4 \pi r^{2} \sigma T^{4}(\sigma$ is Stefan Boltzmann's constant). Taking $r$ from Table 2 (i.e. $\theta / 2$ ) and for $T_{\mathrm{B}}(14 \mu \mathrm{m})=100 \mathrm{~K}$ the corresponding $L(14 \mu \mathrm{m})$ is $6 \times 10^{10} L_{\odot}$ for IC $860,3 \times 10^{10} L_{\odot}$ for Zw049, $2 \times 10^{11} L_{\odot}$ for Arp220W and $8 \times 10^{11} L_{\odot}$ for IRAS 17208 . In terms of temperature this is a lower limit since at $100 \mathrm{~K}$ the excitation of the HCN-VIB line will not be very efficient. We know from the self-absorption structure of $\mathrm{HCN}$ and $\mathrm{HCO}^{+}$(see Sect. 5.1) that there is a temperature gradient and a significant fraction of the HCN-VIB line will emerge from regions of higher temperatures even closer to the nucleus. Thus, when the radius of the emitting region is known - like it is for Arp220W - this value will be a firm lower limit to the real luminosity. For Arp220W, high resolution observations the submm continuum suggest an optically thick, peak dust temperature of 160-200 K (Downes \& Eckart 2007; Sakamoto et al. 2008; Matsushita et al. 2009; Wilson et al. 2014) demonstrating that an assumption of $T_{\mathrm{B}}(14 \mu \mathrm{m})$ of $100 \mathrm{~K}$ is too low. We therefore conclude that these compact mid-IR nuclei may constitute $20-100 \%$ of the total observed $L(I R)$.

From a lower limit to the $T_{\mathrm{B}}(14 \mu \mathrm{m})$ we can estimate an intrinsic (unobscured) $14 \mu \mathrm{m}$ surface brightness, $\Sigma_{14}$. With the assumption of blackbody radiation

$\Sigma_{14}=\frac{L}{2 A}=2 \pi r^{2} \sigma T^{4} / \pi r^{2}=2 \sigma T^{4}$.

For $T_{\mathrm{B}}(14 \mu \mathrm{m})=100 \mathrm{~K}, \Sigma_{14}=3 \times 10^{13} L_{\odot} \mathrm{kpc}^{-2}$, for a $T_{\mathrm{B}}$ $(14 \mu \mathrm{m})$ of $150 \mathrm{~K} \Sigma_{14}=1.5 \times 10^{14} L_{\odot} \mathrm{kpc}^{-2}$ and for $200 \mathrm{~K}$, $\Sigma_{14}=5 \times 10^{14} L_{\odot} \mathrm{kpc}^{-2}$.

Note again, that a more sophisticated approach to the radiative transport and source structure is necessary for a more accurate discussion of the surface brightness and mid-IR luminosity.

\subsubsection{Attenuated dust SEDs}

The luminous mid-IR nucleus, which is exciting the HCN-VIB line, is hidden inside massive layers of dust and its intense emission may become absorbed and re-emitted at longer wavelengths altering the dust spectral energy distribution (SED). The unattenuated SED may be hot (e.g. AGN-like) while the observed SED instead appears cooler and more like that of a typical starburst.

In the conservative $(100 \mathrm{~K})$ estimate of $L(14 \mu \mathrm{m})$ above for IC 860 the unattenuated $14 \mu \mathrm{m}$ continuum flux from the buried source should be $\approx 2.5 \mathrm{Jy}$. However, the observed flux density is more than a factor 30 lower (Lahuis et al. 2007). In Fig. 6 (lower panel) we show an example of a possible intrinsic SED of IC 860 together with the observed SED. Another example is IRAS 17208-0014 where our HCN-VIB observations imply the presence of a $\gtrsim 4$ Jy compact mid-IR source while high resolution imaging at mid-IR wavelengths only finds an extended disk of a total flux of $0.2 \mathrm{Jy}$ (Soifer et al. 2000). The authors note the striking discrepancy between the $2^{\prime \prime}$ mid-IR source and the $0.26 \times 0 . ' 328.6 \mathrm{GHz}$ radio source which sets IRAS 17208-0014 aside from the other ULIRGs they studied. The bright HCNVIB line towards the nucleus of IRAS 17208-0014 suggests that the solution to this discrepancy is an extreme obscuration of the nucleus at mid-infrared wavelengths.

The trapped radiation from an embedded source may also aid in raising the internal temperature significantly. High column densities in all directions lead to the dust being optically thick in the infrared and this radiation is unable to escape and diffuses outwards by multiple absorption/emission events until the dust is optically thin to its own radiation. This process is discussed in Rolffs et al. (2011a) where this "greenhouse effect" (González-Alfonso et al., in prep.) is used to explain the high masses of hot gas near an embedded protostar.

\subsection{The nature of the buried source}

The HCN-VIB lines are emerging from highly compact $(r<17-$ $75 \mathrm{pc}$ ) regions centered on the nucleus of the galaxy. The sphere of influence ${ }^{2}$ of the SMBH in the core of the galaxy is typically $10-20 \mathrm{pc}$. Therefore, the HCN-VIB emission is emerging near to this region and thus originates in a transition-zone constituting an possible evolutionary link between the larger scale host galaxy and the pc-scale environment near the SMBH.

A dust-enshrouded AGN would be consistent with the high mid-IR surface brightness and a temperature gradient causing the $\mathrm{HCN}$ and $\mathrm{HCO}^{+}$self-absorption. The SMBH of IC 860 (for example) would be accreting at 12-30\% of its Eddington limit ${ }^{3}$ to be the cause of the excitation of the HCN-VIB line and $50-100 \%$ of the FIR luminosity. An alternative possibility is that the intense mid-IR emission emerges from a compact starburst. The mid-IR surface brightness of $>5 \times 10^{13} L_{\odot} \mathrm{kpc}^{-2}$ exceeds the limiting Eddington flux of a cool, optically thick starburst. However, a hot, optically thick starburst mode with a bulk temperature $T>200 \mathrm{~K}$ may exist according to Andrews \& Thompson (2011) and they propose that its Eddington flux is $F_{\text {Edd }} \propto 10^{15} L_{\odot} \mathrm{kpc}^{-2}\left(\frac{\Sigma_{g}}{10^{6} M_{\odot} \mathrm{pc}^{-2}}\right)$ (from their Eq. (7) where $\Sigma_{\mathrm{g}}$ is the gas surface density).

For both cases the HCN-VIB line is tracing a phase of exceptional growth in the nucleus of the galaxy in the form of a rapidly accreting SMBH and/or the fast build-up of a nuclear stellar spheriod/disk.

\footnotetext{
2 The region where its gravitational potential overwhelms that of the galaxy with $r_{\mathrm{g}}=2 G M_{\mathrm{BH}} / \sigma_{\mathrm{S}}{ }^{2}\left(\sigma_{\mathrm{S}}\right.$ is the stellar velocity dispersion). It is usually larger than the region inside the Bondi accretion radius $r_{\mathrm{acc}}=2 G M_{\mathrm{BH}} / c_{\mathrm{S}}^{2}$ ( $c_{\mathrm{S}}$ is the speed of sound in the ISM) where the gravitational energy of the SMBH dominates over the thermal energy of the gas.

3 The $K$-band luminosity of IC 860 (Brown et al. 2014) implies a mass of the SMBH of $\approx 2 \times 10^{7} M_{\odot}$ (using the prescription described in Sect. 3.4 of Sakamoto et al. 2013). An SMBH accreting at its Eddington limit would have $L=L_{\mathrm{Edd}}>10^{4.42} M / M_{\odot} L_{\odot}$.
} 

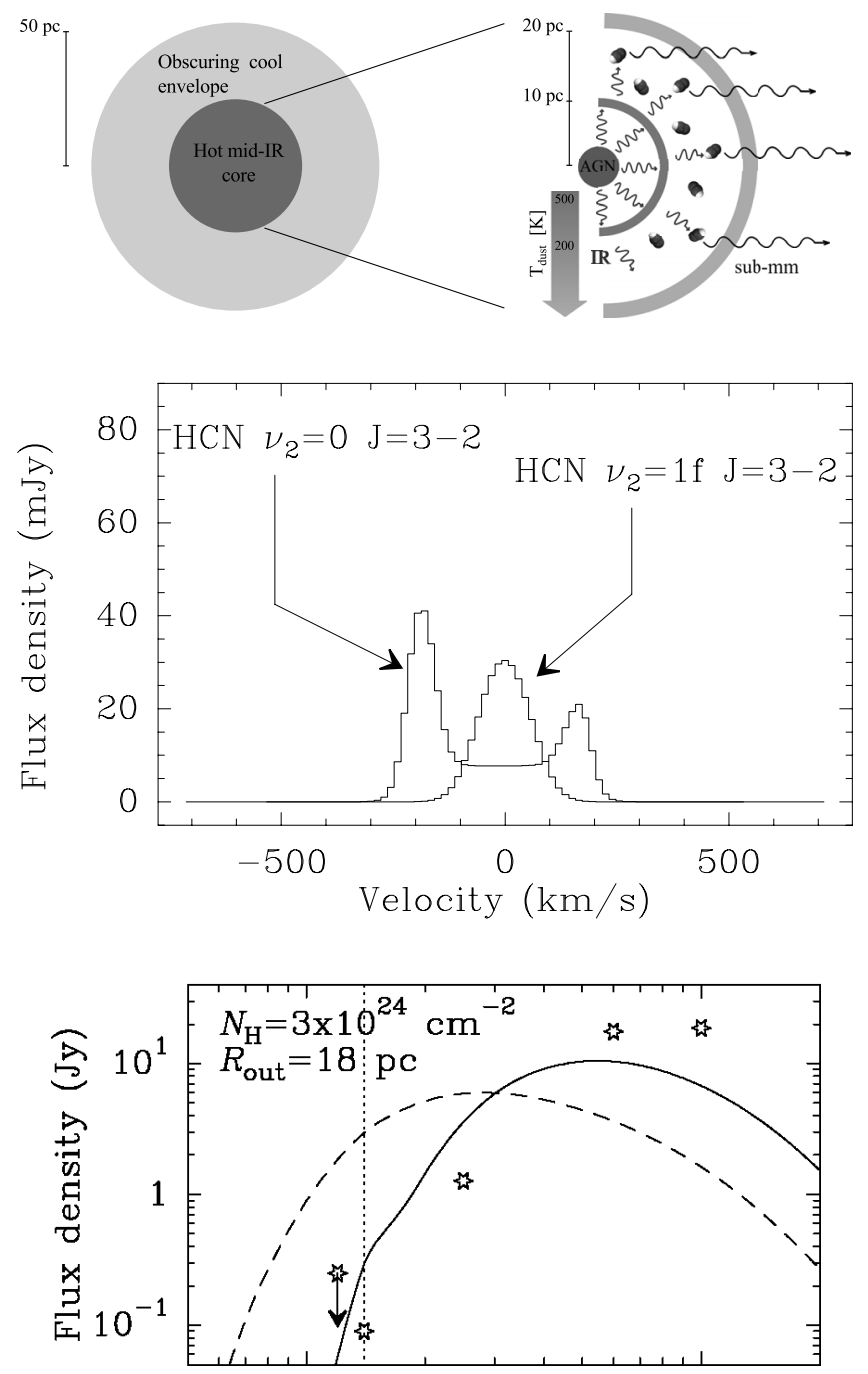

Fig. 6. Upper panel: illustration of the vibrationally excited (HCNVIB) line emission emerging from a buried mid-IR core -surrounded by cooler material giving rise to the self-absorption feature in the $\mathrm{HCN}$ line. An embedded AGN nucleus would be one possible scenario that is powering the bright HCN-VIB and causing the temperature gradient with the resulting self-absorbed $\mathrm{HCN}$ and $\mathrm{HCO}^{+}$lines. Middle panel: illustration of a possible scenario that may account for the observed $\mathrm{HCN}$ and HCN-VIB emission in IC 860 with a buried compact heating source, where the assymmetric $\mathrm{HCN}$ spectrum is caused by an inflow of gas. The model was generated with the tool described in González-Alfonso \& Cernicharo (1999), which includes line overlaps (Gonzalez-Alfonso \& Cernicharo 1997). Note that these examples assume a spherical geometry while our results may imply a more torusor disk-like morphology. Lower panel: this is an illustration of how the dust spectral energy distribution (SED) for IC 860 is shifted from shorter to longer wavelengths by the absorption of the emission from the hot buried source. The dashed curve is the intrinsic unobscured SED and the stars and the solid line indicate the observed flux and the fitted SED.

\subsubsection{A rotating dust torus?}

Interestingly, the HCN-VIB emission of IC 860 and Zw049.057 also shows a faint signature of a twin velocity peak in the $\mathrm{pV}$ diagram (see Fig. 4). This implies that the emission is not arising from a sphere or filled disk but is consistent with a torus-like structure. However, small (pc-scale) optical depth effects in an opaque dust core cannot be excluded, or the possibility that also the HCN-VIB line is partially self-absorbed. The width of the $\mathrm{HCN}-\mathrm{VIB}$ line suggests that the enclosed mass inside $r=18 \mathrm{pc}$

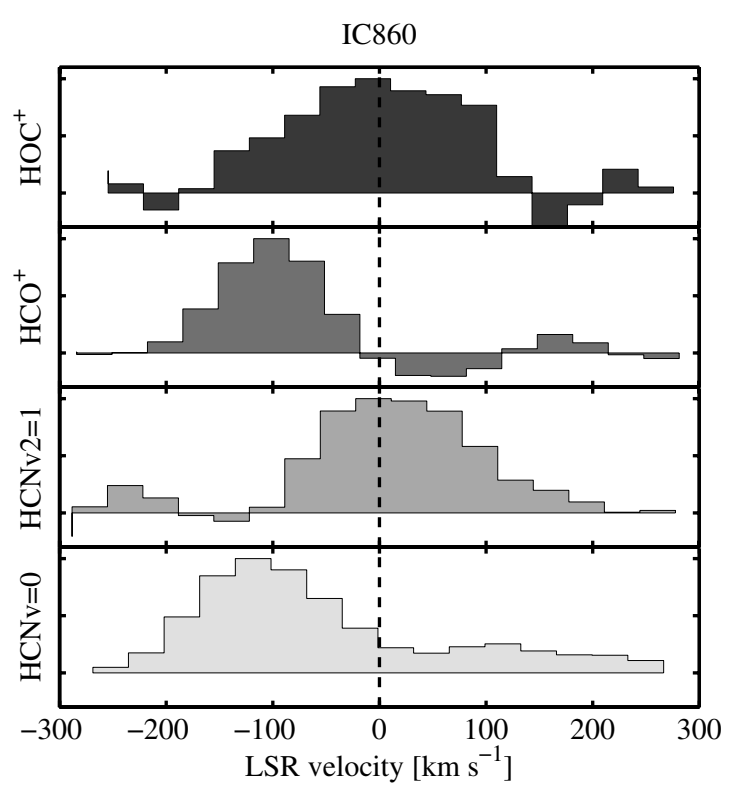

Fig. 7. Zoom in on the spectral structure of $\mathrm{HOC}^{+}, \mathrm{HCO}^{+}$(top two panels) and HCN-VIB, HCN (lower two panels) of IC 860 showing the self-absorbed line shapes of $\mathrm{HCN}, \mathrm{HCO}^{+}$while the other two lines peak at systemic velocity. The intensities have been normalized.

exceeds $5 \times 10^{7} M_{\odot}$ for IC 860 . The $K$-band luminosity implies a mass of the SMBH of $2 \times 10^{7} M_{\odot}$ (see Sect. 5.4) which has error bars of roughly a factor of five. Higher resolution observations of the vibrationally excited $\mathrm{HCN}$ line will allow determinations of the SMBH mass of higher accuracy.

\subsection{Where do we find HCN-VIB emission?}

Even though there has been no systematic survey, the galaxies with HCN-VIB emission detections so far are either ULIRGs or LIRG early type spirals (see Table 4).

The detection of HCN-VIB emission requires the presence of high masses of gas and dust and a compact heating source. Early type spirals have steeper potential wells than late type spirals which may more easily facilitate the formation of compact dust cores. However, optically thick, dusty nuclei should be easier to find in early type spirals than in late type ones since the former generally do not have contaminating star formation in their disks. A survey will reveal whether the HCN-VIB lines preference for early type spirals is real or a result of observational bias, but it is interesting to note that Mignoli et al. (2013) find that moderate luminosity, obscured type-2 AGNs occur primarily in early-type $\mathrm{Sa}-\mathrm{Sb}$ galaxies.

The link between major mergers and ULIRGs is well known (Sanders \& Mirabel 1996; Bekki et al. 1999; Elbaz \& Cesarsky 2003) and luminous, dense circumnuclear disks of gas and stars are a natural consequence of gas-rich galaxy mergers since they trigger massive inflows of gas (Barnes 2002; Mayer et al. 2007). In the very inner region the gas may contribute to the rapid growth of SMBHs and/or the build up of nuclear stellar populations. The incidence of HCN-VIB line emission in ULIRG major mergers will give important clues to when during the ULIRG phase the nuclear region is undergoing accelerated growth. 


\subsubsection{The $L(H C N-V I B) / L(F I R)$ ratio}

In Table 4 we list the $L(\mathrm{HCN}-\mathrm{VIB}) / L(\mathrm{FIR})$ ratio for the eight galaxies that have $\mathrm{HCN}-\mathrm{VIB}$ detections so far: four lenticulars (NGC 1377, NGC 4418, IC 860 and Zw049.057) and four ULIRGs (IRAS 20551-4250, Arp220, Mrk231, IRAS 172080014). We have also added the Galactic massive star formation region SgrB2(M) to illustrate that the ratios for most of the $\mathrm{HCN}$ VIB detected galaxies are higher by an order of magnitude than those of a forming stellar cluster in the centre of the Milky Way. Even if we populated the inner region of (for example) IC 860 solely with SgrB2(M)-like regions this would not reproduce our observations. We have also added the ULIRG IRAS 13120-5453 to Table 4 since we have high signal-to-noise ALMA observations (Privon et al., in prep.) with a good upper limit to the HCNVIB line.

There is no obvious relation between $L(\mathrm{HCN}-\mathrm{VIB}) / L(\mathrm{FIR})$ and FIR luminosity. The ratio will naturally be dependent on the temperature of the exciting dust source and the fraction of the $L$ (FIR) emission that is emerging from the same region as the HCN-VIB emission. However, a trend to be explored further is the correlation with the strength of molecular outflows (see Table 4). The rapidly evolving nature of the studied nuclei is illustrated by what seems to be in- and outflow signatures in the self-absorbed $\mathrm{HCN}$ and $\mathrm{HCO}^{+}$lines, but also in other absorption- and emission lines. All galaxies with a detected HCN-VIB line show evidence of non-circular motions: inflows, outflows or both. Tentatively, the galaxies with the relatively brightest HCN-VIB lines show signs of slow noncircular motions: NGC 4418, Arp220W, IC 860, Zw049.057, IRAS 17208-0014 (see Table 4, for references). (The reversed P-Cygni profiles we find for IC 860 could be evidence of infall, but more observations and modelling are required to determine their precise nature.) Galaxies with fast outflows (i.e. where the outflow velocities exceed the galaxy's rotational (virial) velocity) have fainter HCN-VIB signatures. The ULIRGs Mrk231, IRAS 20551 and IRAS 13120 have prominent molecular outflows, seen in the $\mathrm{OH} 119 \mu \mathrm{m}$ line, with maximum velocities $>1000 \mathrm{~km} \mathrm{~s}^{-1}$ (see Table 4 for references). The lenticular galaxy NGC 1377 has a prominent molecular outflow in relation to its relatively modest escape velocity and mass (Aalto et al. 2012).

Apparently in contrast, Spoon et al. (2013) find, using the $119 \mu \mathrm{m} \mathrm{OH}$ absorption line, that the highest outflow velocities are found in galaxies with deep mid-IR silicate absorption indicating that they are embedded. It is possible that the HCN-VIB line is probing a deeper, more extreme form of nuclear obscuration - and possibly also more transient - than the one traced by the mid-IR silicate absorption. Indeed, Roche et al. (2015) find for NGC 4418 that a large fraction of the silicate absorption is arising from "cool material outside the warm molecular core". In addition, González-Alfonso et al. (2015) find that the silicate absorption is biased towards relatively unabsorbed mid-IR emitting regions and propose high-lying $\mathrm{OH}$ absorption lines as tracers of embeeded, hot nuclei. (The correlation between HCN-VIB and these highly excited $\mathrm{OH}$ lines will be explored in future work.)

A potential scenario is therefore that the HCN-VIB phase peaks right before the nuclear feedback mechanism is fully developed. This would be consistent with our conclusion in Sect. 5.4 that the HCN-VIB line is probing a transient phase of rapid nuclear growth. A possible complication to this picture is the recent discovery of a $800 \mathrm{~km} \mathrm{~s}^{-1} \mathrm{CO} 2-1$ outflow in the HCN-VIB luminous ULIRG IRAS 17208-0014 (Garcia-Burillo et al. 2015). No fast $119 \mu \mathrm{m} \mathrm{OH}$ wind has been found and it is
Table 4. $L(\mathrm{HCN}-\mathrm{VIB})$ vs. $L(\mathrm{FIR})$.

\begin{tabular}{|c|c|c|c|c|}
\hline Name & $\log L(\mathrm{FIR})$ & $\begin{array}{c}\frac{L(\mathrm{HCN}-\mathrm{VIB})}{L(\mathrm{FIR})} \\
{\left[10^{-8}\right]}\end{array}$ & $\begin{array}{c}\text { Molecular } \\
\text { Outflow }^{\dagger}\end{array}$ & Ref. \\
\hline \multicolumn{5}{|l|}{$J=3-2$} \\
\hline NGC 4418 & 11.08 & 3.8 & $n^{\star}$ & [1] \\
\hline IC 860 & 11.17 & 3.2 & $n^{\star}$ & This work \\
\hline Zw 049.057 & 11.27 & 3.4 & $y^{\star}$ & This work \\
\hline Mrk231 & 12.37 & 0.5 & $\mathrm{Y}$ & [2] \\
\hline $\operatorname{SgrB} 2(M)$ & 6.8 & 0.3 & $\mathrm{y}$ & [3] \\
\hline \multicolumn{5}{|l|}{$J=4-3$} \\
\hline NGC 1377 & 10.13 & 1.0 & $\mathrm{Y}$ & [5] \\
\hline NGC 4418 & 11.08 & 9.5 & $n^{\star}$ & [1] \\
\hline IRAS 20551 & 12.00 & 0.4 & $\mathrm{Y}$ & [4] \\
\hline Arp220W & 12.00 & 15 & $\mathrm{y}$ & This work \\
\hline IRAS 13120 & 12.26 & $<0.2$ & $\mathrm{Y}$ & [6] \\
\hline IRAS 17208 & 12.39 & 3.6 & $\mathrm{y}$ & This work \\
\hline
\end{tabular}

Notes. (1) Sakamoto et al. (2010, 2013); (2) Aalto et al. (2015); (3) Rolffs et al. (2011b); (4) Imanishi \& Nakanishi (2013); (5) Aalto et al. (in prep.); (6) Privon et al. (in prep.) ${ }^{(\dagger)} \mathrm{A}$ capital Y indicates molecular outflow velocities exceeding the escape velocity for the nuclear region of the galaxy. References for the outflows/infall: NGC 4418 (González-Alfonso et al. 2012; Sakamoto et al. 2013; Costagliola et al. 2013; Veilleux et al. 2013); IC869 - this work; Zw049.057 Falstad et al. 2015); IRAS 17208-0014 Veilleux et al. (2013; but note recent work by Garcia-Burillo et al. (2015) where CO 2-1 outflow velocities of $800 \mathrm{~km} \mathrm{~s}^{-1}$ are found), Mrk231 (Fischer et al. 2010; Veilleux et al. 2013; González-Alfonso et al. 2014), IRAS 13120-5453 and IRAS 20551 (Veilleux et al. 2013), Arp220W (Sakamoto et al. 2009; Veilleux et al. 2013), SgrB2(m) (Rolffs et al. 2011b). $\left.{ }^{\star}\right)$ Minor axis dust lanes in the form of cones suggesting current or past outflows have been found in NGC 4418 (Sakamoto et al. 2013) and also in IC 860 (unpublished Nordic Optical Telescope U, B images by F. Costagliola). Also in Zw049.057 a peculiar minor axis outflow-like dust feature has been found Scoville et al. (2000).

possible that these absorption studies may miss in- and outflows that occur deep inside an optically thick dust shell.

\subsubsection{HCN-VIB as a survey tool for obscured high-surface brightness activity}

Our observations show that luminous extragalactic HCN-VIB emission is emerging from compact nuclei of high mid-IR surface brightness and that vibrationally excited $\mathrm{HCN}$ is a strong spectral signature of a nucleus in rapid growth - either through Eddington limited (or near-Eddington) nuclear starburst activity or of accreting AGNs. The HCN-VIB line is an effective survey tool to find high surface brightness nuclei hidden behind high masses of cooler dust and gas - even with observations at low spatial resolution. Detecting the vibrationally excited HCN also allows us to reconstruct the intrinsic, hotter SED of the luminosity source.

The HCN-VIB line from a nuclear compact core will show up even if there is more extended star formation which masks and confuses other tracers such as the $[\mathrm{C} \mathrm{II]/FIR} \mathrm{ratio} \mathrm{and} \mathrm{radio}$ continuum imaging. Galaxies with HCN-VIB emission therefore constitute a sample where to search for deeply obscured AGN activity. Any X-ray emission should be severely attenuated even hard $(>5 \mathrm{keV}) \mathrm{X}$-ray emission if column densities exceed $N\left(\mathrm{H}_{2}\right)>10^{24} \mathrm{~cm}^{-2}$. Follow-up, high resolution HCN-VIB and continuum studies will help measure the luminosity density and enclosed mass which will put strong constraints on the nature of the buried source. Thus, studies of vibrationally excited lines can 
be used to reveal and probe a population of deeply dust-buried AGNs promising to shed light on how the SMBH growth connects to that of the stellar bulge and to enable studies of the competition between star formation and accretion. Emerging new techniques utilizing $\mathrm{He}^{+}$recombination lines may further assist in separating accreting SMBHs from hot compact starbursts (Scoville \& Murchikova 2013).

\section{Conclusions}

- High resolution (0.'4) IRAM PdBI and ALMA mm and submm observations of the LIRGs IC 860 and Zw049.057, and the ULIRGs Arp220 and IRAS 17208-0014 reveal luminous vibrationally excited $\mathrm{HCN} J=3-2$ and $4-3 v_{2}=1 \mathrm{f}$ (HCN-VIB) line emission emerging from buried, compact $(r<17-70 \mathrm{pc})$ nuclei. They have very high inferred midinfrared surface brightness $>5 \times 10^{7} L_{\odot} \mathrm{pc}^{-2}$ and are powered by dust enshrouded accreting SMBHs and/or hot (>200 K) extreme starbursts. The HCN-VIB emission may be the signpost of an enshrouded nucleus undergoing rapid evolution either through the growth of its SMBH or a compact stellar spheroid. In either case, the HCN-VIB emission is emerging from inside the inner few tens of pc which consitutes an important transition region and a potential evolutionary link between the larger scale host galaxy disk and the pc-scale environment near a SMBH.

- In contrast, we show evidence that the ground vibrational state rotational lines of $\mathrm{HCN}$ and $\mathrm{HCO}^{+} J=3-2$ and 4-3 (standard tracers of dense gas in galaxies) fail to probe the highly enshrouded, compact nuclear regions due to strong effects of self-absorption (caused by a temperature gradient and large line-of-sight column densities) and absorption of continuum. We advocate HCN-VIB lines as better tracers of the dynamics, mass and physical conditions of (U)LIRG nuclei when $\mathrm{H}_{2}$ column densities exceed $10^{24} \mathrm{~cm}^{-2}$ and when there is a buried compact luminosity source. The vibrationally excited $\mathrm{HCN}$ allows us to identify galaxies where nuclear mid-infrared emission may be strongly absorbed and re-emitted at longer wavelengths - and to reconstruct the intrinsic, hotter dust spectral energy distribution (SED) of the buried luminosity source.

- With this study HCN-VIB lines have now been found in eight (U)LIRGs with AGN signatures and/or evidence of compact $\mathrm{mm} / \mathrm{submm}$ continuum emission. They are either ULIRG mergers or early-type spirals with centrally concentrated molecular gas and dust. The embedded galaxy nuclei show strong signatures of non circular motions that could be interpreted as infall and/or molecular outflows - also in the self-absorbed $\mathrm{HCN}$ and $\mathrm{HCO}^{+}$spectra. IC 860 has reversed P-Cygni profiles that may be evidence of inflowing gas. A tentative conclusion is that galaxies without evidence of fast outflows (i.e. velocities greater than rotational) have the strongest HCN-VIB line in relation to their IR luminosities. This implies that the HCN-VIB emission peaks before the onset of strong feedback.

- All galaxies with detected HCN-VIB lines show very rich molecular spectra and we report the detection of luminous emission lines of $\mathrm{CH}_{2} \mathrm{NH}$ and $\mathrm{HOC}^{+}$and vibrationally excited $\mathrm{HC}_{3} \mathrm{~N}$ in IC 860 and $\mathrm{Zw0} 49.057$ also lines of $\mathrm{CH}_{3} \mathrm{OH}$ are detected. In IRAS 17208-0014, very bright $\mathrm{H}_{2} \mathrm{~S}$ emission is found.

Acknowledgements. We thank the IRAM PdBI staff and the Nordic ALMA ARC node for excellent support. S.A. acknowledges partial support from the
Swedish National Science Council grant 621-2011-4143. K.S. was supported by the MOST grant 102- 2119-M-001-011-MY3. This paper makes use of the following ALMA data: ADS/JAO.ALMA2012.1.00453.S and 2012.1.00817.S.

\section{Appendix A: Self-absorbed $\mathrm{HCN}$ and $\mathrm{HCO}^{+}$lines: Comparing with other species}

\section{A.1. Comparing with the $\mathrm{H}_{2} \mathrm{~S}$ and $\mathrm{CH}_{2} \mathrm{NH}$ lines}

Both the $\mathrm{CH}_{2} \mathrm{NH}$ and $\mathrm{H}_{2} \mathrm{~S}$ molecules have high dipole moments $\left(\mu_{\mathrm{a}}=1.340 \mathrm{D}\right.$ and $\mu=0.97 \mathrm{D}$ respectively) and the transitions observed here have critical densities in excess of $10^{7} \mathrm{~cm}^{-3}$ (Dickens et al. 1997; Crockett et al. 2014). The $\mathrm{CH}_{2} \mathrm{NH} / \mathrm{HCN}$ abundance ratio is typically $10^{-3}-10^{-2}$ (Dickens et al. 1997) suggesting that the $\mathrm{CH}_{2} \mathrm{NH}$ line emission should suffer self-absorption only in extreme conditions. We find that the $\mathrm{CH}_{2} \mathrm{NH}$ line in Zw049.057 has a single peak at systemic velocity and the emission has a maximum at the same spatial position as the HCN-VIB line. Similarly, in IRAS 17208-0014

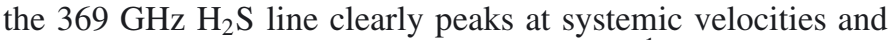
$I\left(\mathrm{H}_{2} \mathrm{~S}\right)>I(\mathrm{HCN})$ between 0 and $-200 \mathrm{~km} \mathrm{~s}^{-1}$. Even though it is possible for the abundances of the two molecules to be similar in the inner regions of hot cores (Crockett et al. 2014) the $\mathrm{H}_{2} \mathrm{~S}$ abundance must be two orders of magnitude greater than that of $\mathrm{HCN}$ for $I\left(\mathrm{H}_{2} \mathrm{~S}\right)>I(\mathrm{HCN})$. This would constitute an hitherto unknown astrochemical scenario. Thus the simplest explanation is that HCN is self-absorbed in the inner region of Zw049.057 and IRAS 17208-0014.

\section{A.2. Comparing with the $\mathrm{HOC}^{+}$line}

Zw049.057 and IC 860 show intensely bright $\mathrm{HOC}^{+} 3-2$ line emission with $I\left(\mathrm{HOC}^{+}\right)>I\left(\mathrm{HCO}^{+}\right)$at systemic velocity (see example for IC 860 in Fig. 7). Assuming the excitation of the two molecules is similar, this either requires that $\mathrm{HOC}^{+}$abundances exceed those of $\mathrm{HCO}^{+}-$or that $\mathrm{HCO}^{+}$is self absorbed. The abundance ratio between the two molecules is a measure of the electron abundance and in Galactic dense clouds $\mathrm{HCO}^{+} / \mathrm{HOC}^{+}$ is typically a few times $10^{3}$. In more extreme regions such as the photon dominated regions (PDRs) of the starburst galaxy M82 and near the nucleus of the Seyfert galaxy NGC 1068, this ratio is found to range between 44 and 1 (Fuente et al. 2008; Usero et al. 2004). In no source has $\mathrm{HOC}^{+}$yet been found to be more abundant than $\mathrm{HCO}^{+}$and chemical models do not find this even in extreme X-ray dominated regions (Spaans \& Meijerink 2007; Bayet et al. 2011). We therefore conclude that $\mathrm{HCO}^{+}$is selfabsorbed in Zw049.057 and in IC 860.

\section{Appendix B: Why does HCN-VIB thrive in obscured regions?}

The HCN $v_{2}=1$ vibrational ladder can commence being excited when $T_{\mathrm{B}}(14 \mu \mathrm{m}) \gtrsim 100 \mathrm{~K}$ (Carroll \& Goldsmith 1981; Aalto et al. 2007). The mid-IR emission must therefore be opaque when the dust temperature is $T_{\text {dust }}=100 \mathrm{~K}$. This requires an optical depth of at least $\tau(14 \mu \mathrm{m}) \gtrsim 5$ and for a standard Galactic extinction curve (Weingartner \& Draine 2001) and $A_{\mathrm{V}} \approx N_{\mathrm{H}} / 2 \times 10^{21} \mathrm{~cm}^{-2}$ this is equivalent to an $\mathrm{H}_{2}$ column density $N\left(\mathrm{H}_{2}\right) \gtrsim 2 \times 10^{23} \mathrm{~cm}^{-2}$. For higher $T_{\text {dust }}$ a lower $\tau$ will be sufficient for $T_{\mathrm{B}}(\mathrm{IR})=100 \mathrm{~K}$ to be satisfied, but at a dust temperature of $400 \mathrm{~K}$ an $\mathrm{H}_{2}$ column density in excess of $=10^{23} \mathrm{~cm}^{-2}$ is still necessary.

The vibrational temperature $T_{\text {vib }}$ is a measure of the $\mathrm{HCN}-\mathrm{VIB}$ excitation temperature and hence of the brightness 
of the background mid-IR radiation field the molecules "see". We can estimate an effective $T_{\text {vib }}$ through comparing the intensities of the HCN-VIB $\left(I_{\mathrm{u}}\right)$ and vibrational ground state lines $\left(I_{1}\right)$ assuming optically thin emission,

$I_{\mathrm{u}} / I_{1}=A_{\mathrm{u}} / A_{\mathrm{l}}\left(v_{\mathrm{u}} / v_{1}\right)^{2} g_{\mathrm{u}} / g_{\mathrm{l}} \exp \left(-E_{\mathrm{u}} / T_{\mathrm{vib}}\right)$.

For a $T_{\text {vib }}$ of $100 \mathrm{~K}$ the optically thin intensity ratio between the HCN-VIB and the HCN line is $3 \times 10^{-5}$ and the HCNVIB line is not detectable. Thus, high column densities are required to observe the HCN-VIB line when $T_{\text {vib }}=100 \mathrm{~K}$. In contrast, for $T_{\mathrm{vib}}=400 \mathrm{~K}$ the optically thin ratio between the $\mathrm{HCN}$ and the HCN-VIB line is 0.08 . However, such a high $T_{\mathrm{B}}$ (mid-IR) requires $T_{\text {dust }}=400 \mathrm{~K}$ combined with a large dust column density for optically thick emission - or a very large $T_{\text {dust }}>400 \mathrm{~K}$. Hence mid-IR excitation of $\mathrm{HCN}$ is favoured in regions of high dust temperature and with significant gas and dust column densities.

\section{References}

Aalto, S., Spaans, M., Wiedner, M. C., \& Hüttemeister, S. 2007, A\&A, 464, 193 Aalto, S., Muller, S., Sakamoto, K., et al. 2012, A\&A, 546, A68

Aalto, S., Garcia-Burillo, S., Muller, S., et al. 2015, A\&A, 574, A85

Andrews, B. H., \& Thompson, T. A. 2011, ApJ, 727, 97

Banerji, M., McMahon, R. G., Hewett, P. C., et al. 2012, MNRAS, 427, 2275

Barnes, J. E. 2002, MNRAS, 333, 481

Bayet, E., Williams, D. A., Hartquist, T. W., \& Viti, S. 2011, MNRAS, 414, 1583

Bekki, K., Shioya, Y., \& Tanaka, I. 1999, ApJ, 520, L99

Brightman, M., \& Ueda, Y. 2012, MNRAS, 423, 702

Brown, M. J. I., Moustakas, J., Smith, J.-D. T., et al. 2014, ApJS, 212, 18

Carroll, T. J., \& Goldsmith, P. F. 1981, ApJ, 245, 891

Cernicharo, J., Agúndez, M., Kahane, C., et al. 2011, A\&A, 529, L3

Condon, J. J. 1997, PASP, 109, 166

Costagliola, F., \& Aalto, S. 2010, A\&A, 515, A71

Costagliola, F., Aalto, S., Rodriguez, M. I., et al. 2011, A\&A, 528, A30

Costagliola, F., Aalto, S., Sakamoto, K., et al. 2013, A\&A, 556, A66

Costagliola, F., Sakamoto, K., Muller, S., et al. 2015, A\&A, 582, A91

Crockett, N. R., Bergin, E. A., Neill, J. L., et al. 2014, ApJ, 781, 114

Díaz-Santos, T., Armus, L., Charmandaris, V., et al. 2013, ApJ, 774, 68

Dickens, J. E., Irvine, W. M., DeVries, C. H., \& Ohishi, M. 1997, ApJ, 479, 307

Downes, D., \& Eckart, A. 2007, A\&A, 468, L57

Downes, D., Solomon, P. M., \& Radford, S. J. E. 1993, ApJ, 414, L13

Elbaz, D., \& Cesarsky, C. J. 2003, Science, 300, 270

Fabian, A. C. 1999, Proc. National Academy of Science, 96, 4749

Falstad, N., González-Alfonso, E., Aalto, S., et al. 2015, A\&A, 580, A52

Fischer, J., Sturm, E., González-Alfonso, E., et al. 2010, A\&A, 518, L41

Fuente, A., García-Burillo, S., Usero, A., et al. 2008, A\&A, 492, 675

Gao, Y., \& Solomon, P. M. 2004, ApJ, 606, 271

Garcia-Burillo, S., Combes, F., Usero, A., et al. 2015, A\&A, 580, A35

Gonzalez-Alfonso, E., \& Cernicharo, J. 1997, A\&A, 322, 938

González-Alfonso, E., \& Cernicharo, J. 1999, ApJ, 525, 845

González-Alfonso, E., Fischer, J., Graciá-Carpio, J., et al. 2012, A\&A, 541, A4

González-Alfonso, E., Fischer, J., Graciá-Carpio, J., et al. 2014, A\&A, 561, A27

González-Alfonso, E., Fischer, J., Sturm, E., et al. 2015, ApJ, 800, 69

Imanishi, M., \& Nakanishi, K. 2013, AJ, 146, 91

Lahuis, F., Spoon, H. W. W., Tielens, A. G. G. M., et al. 2007, ApJ, 659, 296

Lusso, E., Hennawi, J. F., Comastri, A., et al. 2013, ApJ, 777, 86

Malhotra, S., Helou, G., Stacey, G., et al. 1997, ApJ, 491, L27

Martín, S., Krips, M., Martín-Pintado, J., et al. 2011, A\&A, 527, A36

Matsushita, S., Iono, D., Petitpas, G. R., et al. 2009, ApJ, 693, 56

Mayer, L., Kazantzidis, S., Madau, P., et al. 2007, Science, 316, 1874

Medling, A. M. U. V., Guedes, J., et al. 2014, ApJ, 784, 70

Meijerink, R., \& Spaans, M. 2005, A\&A, 436, 397

Merloni, A., Bongiorno, A., Brusa, M., et al. 2014, MNRAS, 437, 3550

Mignoli, M., Vignali, C., Gilli, R., et al. 2013, A\&A, 556, A29

Mills, E. A. C., Güsten, R., Requena-Torres, M. A., \& Morris, M. R. 2013, ApJ, 779,47

Parra, R., Conway, J. E., Aalto, S., et al. 2010, ApJ, 720, 555

Rangwala, N., Maloney, P., Wilson, C., et al. 2015, ApJ, 806, 17
Roche, P. F., Alonso-Herrero, A., \& Gonzalez-Martin, O. 2015, MNRAS, 449, 2598

Rolffs, R., Schilke, P., Wyrowski, F., et al. 2011a, A\&A, 529, A76

Rolffs, R., Schilke, P., Wyrowski, F., et al. 2011b, A\&A, 527, A68

Sakamoto, K., Wang, J., Wiedner, M. C., et al. 2008, ApJ, 684, 957

Sakamoto, K., Aalto, S., Wilner, D. J., et al. 2009, ApJ, 700, L104

Sakamoto, K., Aalto, S., Evans, A. S., Wiedner, M. C., \& Wilner, D. J. 2010, ApJ, 725, L228

Sakamoto, K., Aalto, S., Costagliola, F., et al. 2013, ApJ, 764, 42

Salter, C. J., Ghosh, T., Catinella, B., et al. 2008, AJ, 136, 389

Sanders, D. B., \& Mirabel, I. F. 1996, ARA\&A, 34, 749

Sanders, D. B., Mazzarella, J. M., Kim, D.-C., Surace, J. A., \& Soifer, B. T. 2003, AJ, 126, 1607

Scoville, N., \& Murchikova, L. 2013, ApJ, 779, 75

Scoville, N., Sheth, K., Walter, F., et al. 2015, ApJ, 800, 70

Scoville, N. Z., Evans, A. S., Thompson, R., et al. 2000, AJ, 119, 991

Silk, J., \& Rees, M. J. 1998, A\&A, 331, L1

Soifer, B. T., Neugebauer, G., Matthews, K., et al. 2000, AJ, 119, 509

Spaans, M., \& Meijerink, R. 2007, ApJ, 664, L23

Spoon, H. W. W., Keane, J. V., Tielens, A. G. G. M., et al. 2002, A\&A, 385, 1022

Spoon, H. W. W., Farrah, D., Lebouteiller, V., et al. 2013, ApJ, 775, 127

Treister, E., Urry, C. M., Schawinski, K., Cardamone, C. N., \& Sanders, D. B. 2010, ApJ, 722, L238

Usero, A., García-Burillo, S., Fuente, A., Martín-Pintado, J., \& Rodríguez-Fernández, N. J. 2004, A\&A, 419, 897

Veilleux, S., Meléndez, M., Sturm, E., et al. 2013, ApJ, 776, 27

Weingartner, J. C., \& Draine, B. T. 2001, ApJ, 548, 296

Wilson, C. D., Rangwala, N., Glenn, J., et al. 2014, ApJ, 789, L36

Ziurys, L. M., \& Turner, B. E. 1986, ApJ, 300, L19

1 Department of Earth and Space Sciences, Chalmers University of Technology, Onsala Observatory, 43994 Onsala, Sweden e-mail: saalto@chalmers.se

2 Institut de Radio Astronomie Millimétrique (IRAM), 300 rue de la Piscine, Domaine Universitaire de Grenoble, 38406 St. Martin d'Hères, France

3 Instituto de Astrofísica de Andalucía, Glorieta de la Astronomía, s/n, (IAA-CSIC), 18008 Granada, Spain

4 Universidad de Alcalá de Henares,Departamento de Física y Matemáticas, Campus Universitario, 28871 Alcalá de Henares, Madrid, Spain

5 Institute of Astronomy and Astrophysics, Academia Sinica, PO Box 23-141, 10617 Taipei, Taiwan

6 Jodrell Bank Centre for Astrophysics, School of Physics \& Astronomy, University of Manchester, Oxford Road, Manchester M13 9PL, UK

7 Observatorio Astronómico Nacional (OAN)-Observatorio de Madrid, Alfonso XII 3, 28014 Madrid, Spain

8 Leiden Observatory, Leiden University, 2300 RA, Leiden, The Netherlands

9 Kapteyn Astronomical Institute, University of Groningen, PO Box 800, 9700 AV Groningen, The Netherlands

10 Observatoire de Paris, LERMA (CNRS:UMR8112), 61 Av. de l'Observatoire, 75014 Paris, France

11 Department of Physics and Astronomy, UCL, Gower St., London, WC1E 6BT, UK

12 Argelander-Institut für Astronomie, Auf dem Hügel 71, 53121 Bonn, Germany

13 Infrared Processing and Analysis Center, California Institute of Technology, 1200 East California Boulevard, Pasadena, CA 91125, USA

14 NRAO, 520 Edgemont Road, Charlottesville, VA 22903, USA

15 University of Virginia, Charlottesville, VA 22904, USA

16 Max-Planck-Institut für extraterrestrische Physik, Postfach 1312, 85741 Garching, Germany

17 Group of Molecular Astrophysics, ICMM, CSIC, C/Sor Juana Ines de La Cruz N3, 28049 Madrid, Spain

18 Max-Planck-Institut für Radioastronomie, Auf dem Hügel 69, 53121 Bonn, Germany

19 Astronomy Department, King Abdulaziz University, PO Box 80203, 21589 Jeddah, Saudi Arabia 